\title{
Hybrid atomistic-continuum multiscale method for fluid flow with density variation in microchannels
}

\author{
Van Huyen Vu, Benoît Trouette $\bowtie$, Quy Dong To, Eric Chénier \\ \{van-huyen.vu; benoit.trouette; quy-dong.to;eric.chenier\}@u-pem.fr \\ MSME Laboratoire de Modélisation et Simulation Multi Echelle, \\ UMR 8208 CNRS, Université Paris-Est Marne-la-Vallée, \\ 5 Boulevard Descartes, 77454 Marne-la-Vallée Cedex 2, FRANCE
}

Version: May 19, 2017

\section{Contents}

Nomenclature

1 Introduction 3

2 Modelling and hybrid method 4

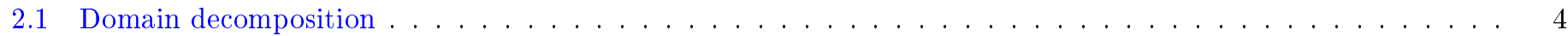

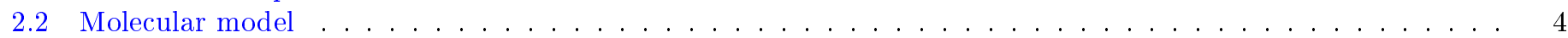

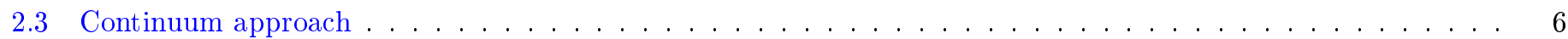

3 Validation, results and discussion 7

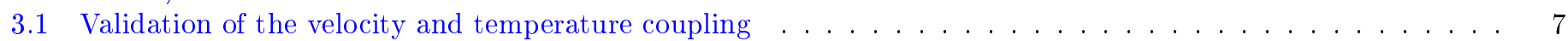

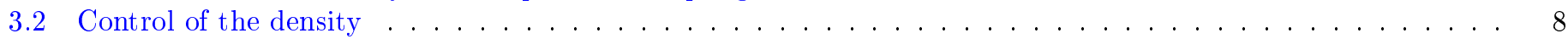

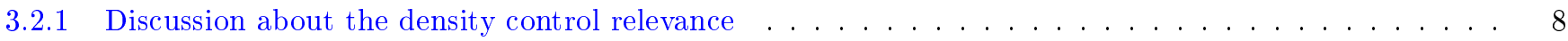

3.2 .2 Algorithm and numerical studies . . . . . . . . . . . . . . . . . . . . . . . . .

3.3 Hybrid multiscale simulations . . . . . . . . . . . . . . . . . . . . . . . . . 13

3.3.1 Compressible flow . . . . . . . . . . . . . . . . . . . . . . . . . 13

3.3.2 Gas flow with condensation at the channel walls . . . . . . . . . . . . . . . . 15

4 Conclusion 19

References 19

\begin{abstract}
The present paper extends the hybrid atomistic-continuum multiscale method developed in Vu et al. (2016) to the study of gas flow problems in long microchannels involving density variations. The simulation domain is decomposed into three regions: the bulk where the continuous Navier-Stokes and energy equations are solved, the neighbourhood of the wall simulated by the Molecular Dynamics and the overlap region which connects the macroscopic variables (density, velocity and temperature) between the two former regions. For the simulation of long micro/nano-channels, a strategy with multiple molecular blocks all along the fluid/solid interface is adopted to capture accurately the macroscopic velocity and temperature variations. The validity of the hybrid method is shown by comparisons with a simplified analytical model in the molecular region. Applications to compressible and condensation problems are also presented and the results are discussed. The hybrid method proposed in this paper allows us a cost-effective computer simulations of large scales problems with an accurate modelling of the transfers at small scales (velocity slip, temperature jump, thin condensation films, ...).

Keywords - Micro/nano-channel - Hybrid simulation - Multiscale flow - Coupled Molecular Dynamics/Finite Volume method - Heat transfer - Interfacial phenomena - Condensation - Compressible flow
\end{abstract}

corresponding author 


\section{Nomenclature}

\section{Roman letters}

$\begin{array}{ll}c_{p}, c_{v} & \text { specific heat at constant pressure and volume, respectively } \\ D & \text { self-diffusion coefficient } \\ \mathbf{e}_{x}, \mathbf{e}_{y}, \mathbf{e}_{z} & \text { Cartesian basis vectors } \\ \mathbf{F} & \text { force } \\ H & \text { channel height } \\ k & \text { stiffness } \\ k_{B} & \text { Boltzmann constant } \\ l, L & \text { channel depth and length } \\ l_{s} & \text { thermal slip length } \\ L_{x}, L_{y} & \text { molecular lengths along the } x, y \text { directions, respectively } \\ m, \bar{m} & \text { masses } \\ n & \text { number of time iterations of density control } \\ n_{b} & \text { number of molecular blocks } \\ N & \text { number of atoms } \\ N_{x}, N_{z} & \text { number of cells along the } x, z \text { directions, respectively } \\ p & \text { pressure } \\ \mathbf{r}, \dot{\mathbf{r}}, \ddot{\mathbf{r}} & \text { microscopic position, velocity and acceleration } \\ r_{b} & \text { scale factor } \\ \mathbf{R} & \text { random force vector } \\ \mathcal{R} & \text { specific gas constant } \\ T & \text { temperature } \\ \mathbf{u} & \text { macroscopic velocity } \\ u, v, w & \text { velocity components along the } x, y \text { and } z \text { directions, respectively } \\ U & \text { internal energy } \\ V & \text { potential } \\ x, y, z & \text { Cartesian coordinates } \\ Z & \text { compressibility factor }\end{array}$

Greek symbols

$\begin{array}{ll}\alpha & \text { coefficient } \\ \delta t, \Delta t & \text { microscopic and macroscopic time steps } \\ \delta & \text { condensation thickness } \\ \Delta z & \text { space step of the molecular region in the } z \text {-direction } \\ \varepsilon & \text { potential well-depth } \\ \lambda & \text { thermal conductivity } \\ \mu & \text { dynamic viscosity } \\ \rho & \text { density } \\ \sigma & \text { potential "diameter" } \\ \tau & \text { reference time scale } \\ \overline{\bar{\tau}} & \text { viscous stress tensor } \\ \xi & \text { coefficient }\end{array}$

\section{Subscripts}

$\begin{array}{ll}C & \text { continuum } \\ f & \text { fluid } \\ g & \text { gas phase } \\ \text { in } & \text { inlet } \\ l & \text { liquid phase } \\ M & \text { molecular } \\ O & \text { overlap } \\ w & \text { wall }\end{array}$

Superscripts

anal

analytical 


\section{Introduction}

The miniaturization technology enables the design of compact heat exchangers and improves the performance of the device by increasing heat transfer area. However, reducing the characteristic dimensions of the system also gives rise to new physical phenomena related to the fluid/solid molecular interactions. At the micro/nano scale, conventional hydrodynamics equations may be no longer valid and interface effects like velocity slip and temperature jump become significant. New fluid models and boundary conditions have been developed to account for these effects but they are still subject to numerous limitations (Dadzie and Méolans, 2005; Struchtrup, 2013; To et al., 2015). For example, they require unknown input parameters such as the accommodation coefficients and can not capture all the surface physic complexity like Knudsen layer, adsorption/desorption, evaporation/condensation, etc.

To enhance the description of the fluid flow in channels with large aspect ratio, a hybrid numerical model coupling an atomistic approach and a continuum model has been recently developed to take into account the multiscale behaviour of the transfer phenomena ( $\mathrm{Vu}$ et al., 2016). The Molecular Dynamics simulation method is used in molecular blocks distributed along the channel wall, while a Finite-Volume discretization is adopted in the flow bulk to solve the macroscopic transfer equations in the axial direction of the channel. The purpose of this paper is to extend our hybrid atomistic-continuum method recently proposed for incompressible and compressible fluid flows to physical problems involving density variations.

Over the last decades, hybrid methods have been developed to combine the benefits of different approaches to investigate fluid flow problems in geometries with very different characteristic length scales. They allow a tailored approach to the consideration of both the small and the large length scales of the system, modelled with microscopic and macroscopic approaches respectively, for an optimal computational cost. The macroscopic part is modelled with continuum mechanics where fundamental physical laws such as the conservation of mass, the conservation of momentum, and the conservation of energy verify. The most commonly used model is the the Navier-Stokes and energy equations. However, if the wall effects are dominant or if the constitutive relation is unknown, the Navier-Stokes and energy equations are no longer practicable. A microscopic model is then be used to describe the missing parts of the macroscopic approach, especially near the wall boundary. In most cases, molecular dynamics is used to describe the microscopic scale. However, its prohibitive cost allows only the simulation of domains of few nanometres over few picoseconds and it is more suitable for homogeneous systems via the use of periodic boundary conditions. In addition to the methodological and fundamental developments (see Mohamed and Mohamad, 2010, for a full literature review), they have been successfully applied to the study of micro- and nano channels of high aspect ratio (Borg et al., 2013b; Vu et al., 2016), including condensation (Sun et al., 2009), moving contact-line problem (Hadjiconstantinou, 1999), flow around carbon nano tube (Werder et al., 2005; Dupuis et al., 2007), singularity of continuum model (Nie et al., 2004) as well as nanofluidic networks of arbitrary complexity (Stephenson et al., 2015).

Three classes of methods exist in the literature. Historically, the first one is the Domain Decomposition Method (DDM) by O'Connell and Thompson (1995). The molecular dynamics approach is used to describe fluid/walls interaction in micro channels while the incompressible Navier-Stokes equations are solved in the remote region. Primary variables or fluxes are synchronized between the two approaches and an overlap region ensures their spatial continuity. Various approaches for applying macroscopic boundary conditions to microscopic simulations have been investigated (Drikakis and Asproulis, 2010). To deal with non periodic conditions, a boundary volume force is generally added (Zhou et al., 2014) to reduce the stratification issue at boundaries. Initially, the DDM dealt only with dynamical problems, but recent developments have incorporated the thermal effects (Liu et al., 2007).

A second approach is the Heterogeneous Multiscale Method (HMM) introduced by Ren and E (2005). The domain is fully described by a macroscopic approach and microscopic simulations are used in different regions of the domain to locally estimate the needed quantities of the macroscopic solver. As for the DDM, the micro resolutions can be placed at the walls to estimate the slip properties but they also can be arranged in the core flow to assess macroscopic coefficients as the viscosity or conductivity or to obtain the stress tensor if no constitutive law is assumed. Contrary to the DDM, it does not allow the temporal coupling between the time scales.

Recently, Borg et al. (2013b) and Patronis et al. (2013); Borg et al. (2015) developed the Internal-flow Multiscale Method (IMM) for incompressible and compressible flows respectively. Their approach is dedicated to flows in nano channels where wall effects are predominant. The hydraulic diameter of the channels is then limited to a few nanometres. An important point is that no constitutive law is assumed and the various algorithms implemented are based on the conservation of mass and the momentum. Furthermore, the microscopic region can always be chosen to keep periodic boundary conditions. On the other hand, the scheme is limited to dealing with the mass conservation and the heat transfer issue is totally neglected. The IMM has also been used for complex geometries or nanofluidic networks (Borg et al., 2013a; Stephenson et al., 2015). 
Given the small size of the systems, this method allows, at least in the simplest case, comparisons with pure molecular dynamics simulations. The fact of not requiring sliding properties, relationship on the stress tensor but also keeping periodic boundary conditions makes this approach simple to implement but in return it is difficult to deal with thermal problems.

In our approach, the domain decomposition approach is used, but similarly to the IMM and HMM methods, the vicinity of the wall is discretized in micro elements. In this way the large aspect ratio micro and nano channels can be simulated (Vu et al., 2016); the information transfer from microscopic fields to continuous field is done by interpolations between the micro simulations. This approach keeps the benefits of domain decomposition method, i.e. thermal effect can be simulated, for a limited computational cost: a wall of few millimetre length is substituted by some micro element simulation of nanometre length. Particular attention is paid to control the density in each micro simulation.

The paper is organized as follow. After this introduction, the hybrid and multiscale methods are detailed in Sec. $2 . \quad$ The results are detailed in Sec. 3. The hybrid method is first validated for a single molecular block on the transient Couette and heat conduction problems. Next, we tackle the issue of the density variation in the simulation by proposing a special density control algorithm. The algorithm is then applied in hybrid multiscale simulations to solve fluid flows and heat transfer in long narrow channels. Finally, some conclusions and remarks are given at the end of the paper.

\section{Modelling and hybrid method}

A channel of rectangular section of height $2 H$, depth $l \gg 2 H$ and length $L \gg l$ is considered in this paper. The inner walls are made of platinum and argon in liquid or gaseous phase is injected through the inlet section. The walls, located at $y= \pm l / 2$, are thermally isolated whereas those at $z=0$ and $2 H$ are kept at the constant temperature $T_{w}$. Considering the aspect ratio $l / 2 H \gg 1$ and the adiabatic boundary conditions at $y= \pm l / 2$, it is relevant to adopt a two-dimensional model. Since the flow is symmetric about the mi-plane $z=H$, the simulation domain can be halved to reduce the computational cost.

\subsection{Domain decomposition}

The decomposition domain method herein above mentioned is adapted in this work to the study of high aspect ratio narrow channels. A Multi-DDM scheme using different molecular blocks is adopted (Fig. 1). The methodological and numerical aspects have been wildly detailed in Vu et al. (2016) and references herein; only the main features are recalled here. The rectangular geometry of the channel is divided into three sub-domains:

1. In the bulk of the channel, above the dashed line in Fig. 1, the fluid flow and heat transfer are governed by the continuum theory. This region of length $L$, height $H_{C}$ and label $(\mathrm{C})$ is devoted to simulating the fluid at the macroscale.

2. In the molecular/atomistic regions, $\mathrm{M}_{i}(i=1, \ldots, 4$ in Fig. 1), the particle approach is used to model the fluid/solid interactions at the micro-/nanoscales. Figure 2(a) is an enlargement of one of the numbered rectangular blocks drawn in Fig. 1.

3. The overlap region of height $H_{O}$ (Fig. 2(b)) is designed to enable the communication between the continuum domain and the different molecular blocks. The size of this overlap zone is $40 \%$ of $H_{M, f}$ such as $H=H_{C}+0.6 H_{M, f}$. It is divided into 4 sub-layers: two of them guarantee the exchanges between the continuum and molecular domains $(\mathrm{C} \rightarrow \mathrm{M}$ and $\mathrm{M} \rightarrow \mathrm{C}$ ), a relaxation layer allows decoupling the dynamic between the two previous regions and a control layer serves as a buffer region to minimize spurious effects caused by the upper specular wall. In this paper, the control layer is also used for controlling the number of atoms in the molecular block.

\subsection{Molecular model}

In each molecular block, the Lennard-Jones potential is used to describe the pair interactions argon/argon for fluid/fluid and argon/platinum for fluid/wall interactions. Given two atoms of type $\alpha$ and $\beta$ at distance $r$, the potential $V_{\alpha \beta}(r)$ of the pair reads

$$
V_{\alpha \beta}(r)=4 \varepsilon_{\alpha \beta}\left[\left(\frac{\sigma_{\alpha \beta}}{r}\right)^{12}-\left(\frac{\sigma_{\alpha \beta}}{r}\right)^{6}\right]
$$

where the subscript $\alpha \beta$ stands for $f f$ (fluid/fluid) or $f w$ (fluid/wall). The parameters $\varepsilon_{\alpha \beta}$ and $\sigma_{\alpha \beta}$ are respectively the potential well-depth and the "diameter". Taking argon as the reference material, the potential parameters for the couples $\mathrm{Ar} / \mathrm{Ar}, \mathrm{Ar} / \mathrm{Pt}$ and their atomic masses $m_{f}$ and $m_{w}$ can be expressed in the reduced Lennard-Jones units: $\varepsilon_{f f}=\varepsilon, \sigma_{f f}=\sigma$, $m_{f}=\bar{m}, \varepsilon_{f w}=0.953 \varepsilon, \sigma_{f w}=0.906 \sigma, m_{w}=4.8833 \bar{m}$ with $\varepsilon=1.656 \times 10^{-21} \mathrm{~J}, \sigma=3.405 \AA$ and $\bar{m}=6.633 \times 10^{-26} \mathrm{~kg}$. The reference scales for time, temperature and velocity are $\tau=\sqrt{\bar{m} \sigma^{2} / \varepsilon}=2.155 \times 10^{-12} \mathrm{~s}, \varepsilon / k_{B}=120 \mathrm{~K}$ and $\sqrt{\varepsilon / \bar{m}}=158.03$ $\mathrm{m} / \mathrm{s}$, respectively. With these basic reference scales, the density, pressure, viscosity, conductivity and specific heat are $\bar{m} / \sigma^{3}$, 


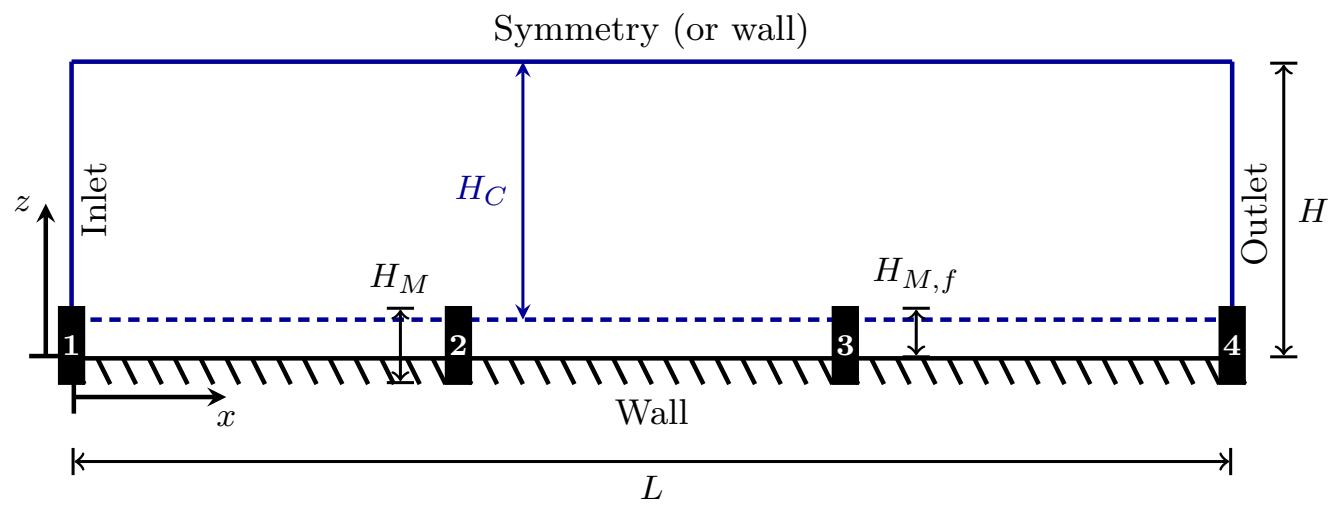

Figure 1: Sketch of a hybrid multiscale domain of height $H$ and length $L$. The continuum region (height $H_{C}$ ) is governed by the Navier-Stokes and energy equations. An atomistic description is used to model the wall and the adjacent fluid in the numbered rectangular blocks of height $H_{M}=H_{M, w}+H_{M, f}$ (Fig. 2(a)).

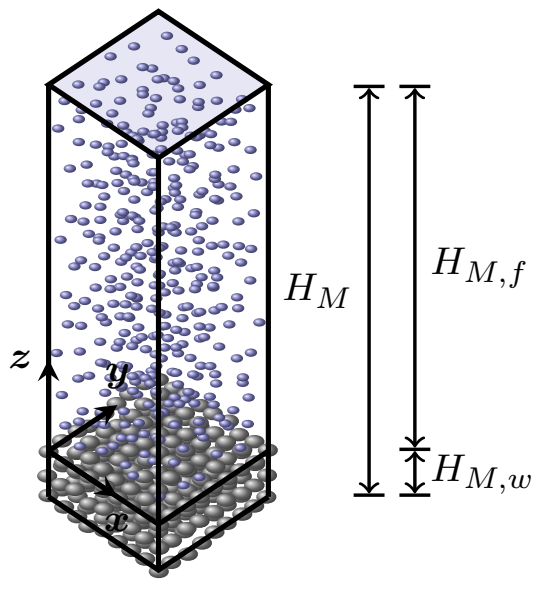

(a)

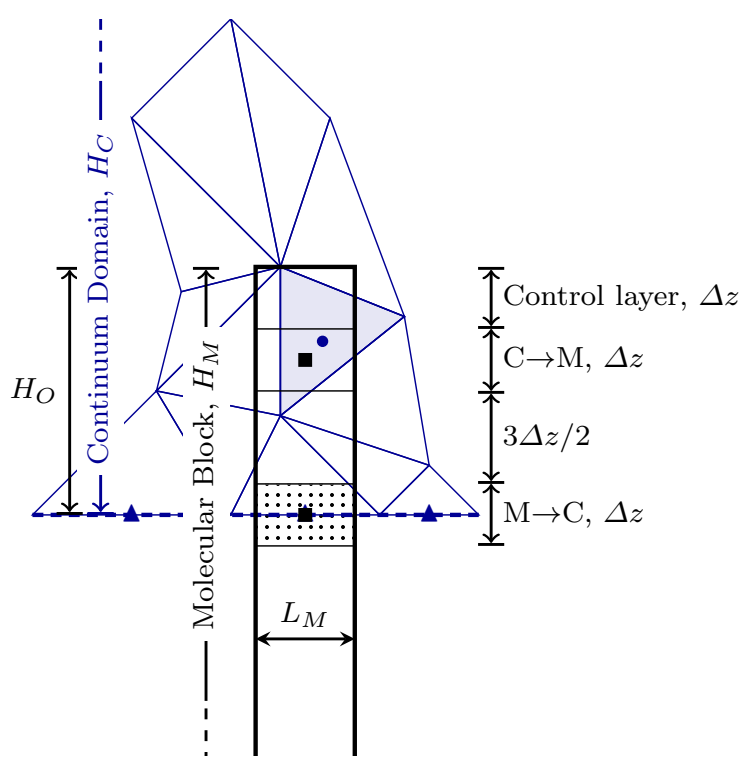

(b)

Figure 2: (a) Molecular block of simulation of total height $H_{M}=H_{M, f}+H_{M, w}$, where $H_{M, f}$ is the fluid height and $H_{M, w}$ the wall thickness. The bluish top surface stands for a specular wall. (b) Overlap region of length $H_{O}$ which ensures the communication between the molecular domain of dimensions $L_{M} \times H_{M}$ and the continuum domain, here partially covered by a mesh of triangles. The $\mathrm{M} \rightarrow \mathrm{C}$ and $\mathrm{C} \rightarrow \mathrm{M}$ layers enable the transfer of the macroscopic or averaged variables, between the molecular and continuum parts. The intermediary layer of size $3 \Delta z / 2$ is a buffer zone between the two previous layers. The control layer is a buffer zone which is also used for inserting or removing particles in the molecular domain. The value $\Delta z$ is typically $10 \%$ of $H_{M, f}$. Blue symbols are Finite Volume nodes (triangles at the boundary and filled circle in the core domain). The black square in the dotted rectangle is a Finite Volume node on which the averaged values in the $\mathrm{M} \rightarrow \mathrm{C}$ layer are calculated. Reciprocally, Finite Volume extrapolations for the temperature and velocity are expressed at the centre of the $\mathrm{C} \rightarrow \mathrm{M}$ layer (second black square) in order to drive the microscopic quantities to their macroscopic counterparts.

$\varepsilon / \sigma^{3}, \sqrt{\bar{m} \varepsilon} / \sigma^{2}, k_{B} \sqrt{\bar{m} \varepsilon} / \sigma^{2}$ and $k_{B} / \bar{m}$, respectively. Later in this paper, variables will be given, unless otherwise stated, in Lennard-Jones reduced units.

The solid walls are modelled with three layers of platinum atoms arranged in a $\mathrm{FCC}(111)$ lattice with density $\rho_{w}=12.76$. Each solid atom is connected to its nearest neighbours with springs of stiffness $k=3249.1 \varepsilon / \sigma^{2}$ which generates the harmonic potential $V_{w w}(r)=k r^{2} / 2$. Two extra layers of "phantom atoms" are added below the three layers in order to keep the wall stationary and to impose the desired temperature $T_{w}$ (Maruyama and Kimura, 1999; Maruyama, 2000).

With the potentials and the current positions $\mathbf{r}_{1}, \ldots, \mathbf{r}_{N}$ of all the $N$ fluid and solid atoms of the system being known, we 
are able to determine the force $\mathbf{F}_{i}$ applied to any atom $i$ and its acceleration $\ddot{\mathbf{r}}_{i}$ according to Newton's second law:

$$
m_{i} \ddot{\mathbf{r}}_{i}(t)=\mathbf{F}_{i}(t) \stackrel{\text { def }}{=} \frac{\partial}{\partial \mathbf{r}_{i}} V_{t o t}\left(\mathbf{r}_{1}, \ldots, \mathbf{r}_{N}\right)
$$

where $V_{\text {tot }}$ denotes all the possible interactions (i.e. fluid/fluid, fluid/wall or wall/wall):

$$
V_{t o t}\left(\mathbf{r}_{1}, \ldots, \mathbf{r}_{N}\right)=\frac{1}{2} \sum_{i, j \neq i} V_{f f}\left(\left\|\mathbf{r}_{i}-\mathbf{r}_{j}\right\|\right)+\frac{1}{2} \sum_{i, j \neq i} V_{f w}\left(\left\|\mathbf{r}_{i}-\mathbf{r}_{j}\right\|\right)+\frac{1}{2} \sum_{i, j \neq i} V_{w w}\left(\left\|\mathbf{r}_{i}-\mathbf{r}_{j}\right\|\right)
$$

A volumetric force $\mathbf{F}_{e x t}$ is also applied to the fluid particles to model the pressure contribution $\partial_{x} p$ coming from the continuum region and directed along $x$ (direction vector $\mathbf{e}_{x}$ ): $\mathbf{F}_{e x t}=-m_{f} \partial_{x} p / \rho \mathbf{e}_{x}$. The temporal integration of the motion equations is performed with the Velocity-Verlet algorithm. The microscopic time step is $\delta t=5 \times 10^{-3}$. From a practical point of view, it is not necessary to treat all pair interactions: the Verlet list is adopted and the contributions of pairs in $V_{t o t}$ with distances higher than the cut-off radius $r_{c}=2.5$ are neglected in the force computation (Allen and Tildesley, 1989; Rapaport, 2004).

Periodic boundary conditions are applied in $x$ - and $y$-directions leading to a zero mass flux in the vertical direction. In order to keep the wall at the desired temperature $T_{w}$, a force $\mathbf{F}_{i, w}$ is introduced in the equation of motion for the solid particles of the upper phantom layer. Using the Langevin thermostat (Allen and Tildesley, 1989), this force reads

$$
\mathbf{F}_{i, w}=-\alpha_{w} \dot{\mathbf{r}}_{i}+\mathbf{R}_{i, w}\left(T_{w}\right)
$$

with $\alpha_{w}=168.3 \tau^{-1}$ and $\mathbf{R}_{i, w}$ a random force vector. Each independent component of $\mathbf{R}_{i, w}$ is sampled from a Gaussian distribution with a zero mean and a standard deviation $\sqrt{2 \alpha_{w} m_{w} k_{B} T_{w} / \delta t}$ depending on the prescribed temperature $T_{w}$.

In the $\mathrm{C} \rightarrow \mathrm{M}$ layer, both dynamic and temperature must be controlled in a way that the mean velocity $\langle\dot{\mathbf{r}}\rangle(z)$ and the mean temperature $\left\langle\bar{m}[\dot{\mathbf{r}}(z)-\langle\dot{\mathbf{r}}\rangle(z)]^{2}\right\rangle / 3 k_{B}$ coincide with the local linear macroscopic approximation of the velocity $\mathbf{u}_{C \rightarrow M}(z)$ and temperature $T_{C \rightarrow M}(z)$ determined from the continuous model (Sun et al., 2012). To this end, Newton's equations are first solved with an additional force in the $\mathrm{C} \rightarrow \mathrm{M}$ layer so that the mean velocity of the fluid atoms is constrained to the macroscopic velocity profile:

$$
m_{f} \ddot{\mathbf{r}}_{i}^{\star}=\mathbf{F}_{i}(t)-\langle\mathbf{F}(t)\rangle+\xi \frac{m_{f}}{\delta t}\left(\mathbf{u}_{C \rightarrow M}(z)-\langle\dot{\mathbf{r}}(t)\rangle(z)\right)
$$

where $\langle\phi\rangle$ denotes an averaged value of the variable $\phi$ and $\langle\phi\rangle(z)$ refers to the variation of $\langle\phi\rangle$ with respect to the $z$ ordinate (Vu et al., 2016). The intermediate acceleration $\ddot{\mathbf{r}}_{i}^{\star}$ computed previously is then corrected via a Langevin thermostat, to control the temperature:

$$
m_{f} \ddot{\mathbf{r}}_{i}(t)=m_{f} \ddot{\mathbf{r}}_{i}^{\star}(t)-m_{f} \alpha\left(\dot{\mathbf{r}}_{i}(t)-\langle\dot{\mathbf{r}}(t)\rangle(z)\right)+\mathbf{R}_{i, f}\left(T_{C \rightarrow M}\right)
$$

Like the solid thermostat, each independent component of the random force vector $\mathbf{R}_{i, f}$ is sampled from a Gaussian distribution with a standard deviation build on the continuum temperature: $\sqrt{2 \alpha m_{f} k_{B} T_{C \rightarrow M} / \delta t}$. The parameters in Eqs. (5) and (6) are $\alpha=1 \tau^{-1}$ and $\xi=5 \times 10^{-3}$ or $2 \times 10^{-2}$ depending on the fluid is in a gaseous or liquid phase.

The coupling scheme is based on the exchange of the primary variables. For the molecular (M) to continuum (C) coupling, the macroscopic components $\mathbf{u}_{M \rightarrow C}=\langle\dot{\mathbf{r}}\rangle$ and $T_{M \rightarrow C}=\left\langle\bar{m}\left[\dot{\mathbf{r}}-\mathbf{u}_{M \rightarrow C}\right]^{2}\right\rangle / 3 k_{B}$ are computed into the M $\rightarrow \mathrm{C}$ layer centered around the lower boundary of the continuum region (Fig. 2(b)). By construction, this scheme guarantees the continuity of the primary variables, and also their respective fluxes if the macroscopic properties are the same in the two domains (molecular and continuum) over the overlap region. The density stemming from the two models must also be identical. To this aim, a control method for the density is often required (see Sec. 3.2.2 for the algorithm presentation).

\subsection{Continuum approach}

In the continuum region, the Navier-Stokes and energy equations (Bird et al., 2006) are used, coupled with an equation of state linking the density, the pressure and the temperature:

$$
\left\{\begin{array}{l}
\frac{\partial \rho}{\partial t}+\nabla \cdot(\rho \mathbf{u})=0 \\
\frac{\partial \rho \mathbf{u}}{\partial t}+\nabla \cdot(\rho \mathbf{u} \otimes \mathbf{u})=-\nabla p+\nabla \cdot \overline{\bar{\tau}} \\
c_{p}\left(\frac{\partial \rho T}{\partial t}+\nabla \cdot(\rho \mathbf{u} T)\right)=\nabla \cdot(\lambda \nabla T)-\left(\frac{\partial \ln \rho}{\partial \ln T}\right) \frac{D p}{D t}+\overline{\bar{\tau}}: \nabla \mathbf{u} \\
f_{\operatorname{eos}}(p, \rho, T)=0
\end{array}\right.
$$


where $\mathbf{u}=u \mathbf{e}_{x}+w \mathbf{e}_{z}, p$ and $T$ are the two dimensional velocity, the pressure and the temperature fields; $\overline{\bar{\tau}}$ is the viscous stress tensor; $\rho, \mu, \lambda$ and $c_{p}$ are the fluid density, the dynamic viscosity, the thermal conductivity and the specific heat. The equation of state of Kolafa and Nezbeda (1994), fitted from molecular dynamics simulations of a Lennard-Jones fluid, is adopted. It provides an analytical expression built on the compressibility factor $Z$ which is function of density and temperature:

$$
f_{\text {eos }} \stackrel{\text { def }}{=} p-Z(\rho, T) \rho \mathcal{R} T
$$

with $\mathcal{R}$ the specific gas constant. When $Z=1$, we recover the equation of state for ideal gases.

As mentioned in the previous section, the continuity of the momentum and energy fluxes between the atomistic and continuum domains is ensured if the density $\rho$, the transport coefficients $\mu, \lambda$ and the specific heat $c_{p}$ are identical in the overlap region. Based on the macroscopic knowledge of the pressure and temperature, the density is calculated in the continuum domain by solving the non-linear equation $(7 \mathrm{~d})$. The dynamic viscosity and the thermal conductivity are evaluated from the correlations by Galliéro et al. (2005) and Bugel and Galliéro (2008), respectively. The specific heat at constant pressure $c_{p}$ and volume $c_{v}$ are related by classical thermodynamics laws:

$$
c_{p}-c_{v}=\frac{T(\partial p / \partial T)^{2}}{\rho^{2}(\partial p / \partial \rho)} \quad \text { with } \quad c_{v}=\frac{\partial U}{\partial T}
$$

where $U$ is the internal energy which consists of two contributions, one is provided by the ideal gas law and the second is a corrective term given by Kolafa and Nezbeda (1994). The partial derivatives in Eq. (9) are computed from the analytical equation of state Eq. (8).

For the continuum domain, the lower boundary conditions are derived from the molecular blocks:

$$
\text { at } z=z_{M \rightarrow C}:\left\{\begin{array}{l}
\mathbf{u}(x)=u_{M \rightarrow C}^{\operatorname{macro}}(x) \mathbf{e}_{x} \\
T(x)=T_{M \rightarrow C}^{\operatorname{macro}}(x)
\end{array}\right.
$$

where $z_{M \rightarrow C}$ is the ordinate of the lower bound of the continuum domain which coincides with the centre of the $\mathrm{M} \rightarrow \mathrm{C}$ layer of the molecular region (Fig. 2(b)). The two terms $u_{M \rightarrow C}^{\text {macro }}(x)$ and $T_{M \rightarrow C}^{\text {macro }}(x)$ are piecewise linear functions. Denoting $u_{M_{i} \rightarrow C}^{\text {macro }}$ the mean velocity of particles in the $\mathrm{M}_{i} \rightarrow \mathrm{C}$ layer of the $i$-th molecular block $\mathrm{M}_{i}$, the macroscopic velocity reads:

$$
u_{M \rightarrow C}^{\operatorname{macro}}(x)=\sum_{i=1}^{n_{b}-1}\left(u_{M_{i} \rightarrow C}^{\operatorname{macro}}+\frac{u_{M_{i+1} \rightarrow C}^{\text {macro }}-u_{M_{i} \rightarrow C}^{\text {macro }}}{x_{M_{i+1} \rightarrow C}-x_{M_{i} \rightarrow C}}\left(x-x_{M_{i} \rightarrow C}\right)\right) \times \mathcal{H}\left(x, x_{M_{i+1} \rightarrow C}, x_{M_{i} \rightarrow C}\right)
$$

where $x_{M_{i} \rightarrow C}$ (resp. $x_{M_{i+1} \rightarrow C}$ ) is the abscissa of the centre of the $\mathrm{M}_{i} \rightarrow \mathrm{C}$ (resp. $\left.\mathrm{M}_{i+1} \rightarrow \mathrm{C}\right)$ layer, $\mathcal{H}(x, a, b)=H(a-x) \times$ $H(x-b)$ with $H(x)$ the Heaviside step function, and $n_{b}$ is the number of molecular blocks. The same approximation is applied for the temperature profile $T_{M \rightarrow C}^{\operatorname{macro}}(x)$.

At the upper border $z=H$, either the velocity and the temperature are imposed or a symmetry condition is prescribed $\left(\partial_{z} u=w=0\right.$ and $\left.\partial_{z} T=0\right)$. Two types of conditions are considered for the inlet/outlet sections of the channel. A pressure drop may be imposed throughout the channel or the velocity and temperature profiles may be assigned at the entrance. In each of these two cases, the fluid flow and heat transfer are assumed fully developed at the outlet: $\partial_{x} u=w=0$ and $\partial_{x} T=0$. For purely hybrid simulations, namely with a single molecular block $\left(n_{b}=1\right)$, Eq. (10) is no longer relevant. The velocity and temperature expressions are simply substituted by $u_{M \rightarrow C}^{\text {macro }}(x)=u_{M_{1} \rightarrow C}^{\text {macro }}$ and $T_{M \rightarrow C}^{\text {macro }}(x)=T_{M_{1} \rightarrow C}^{\text {macro }}$. This configuration is suitable for the study of one-dimensional problems which permits us the validation of the hybrid method (Couette or Poiseuille flows in Sec. 3.1) and the study of the density coupling algorithm (Sec. 3.2).

The solutions of the Navier-Stokes and energy equations (7) are approximated with a second order Finite Volume scheme with collocated variables and unstructured meshes (Chénier et al., 2006, 2008; Touazi et al., 2008). The non-linear set of equations is solved iteratively with a Newton-Raphson algorithm.

\section{Validation, results and discussion}

\subsection{Validation of the velocity and temperature coupling}

In this section, the hybrid approach is illustrated for a single molecular block on the transient Couette and conduction problems. For the Couette problem, the two walls at $z=0$ and $z=H$ are kept at the constant temperature $T_{1}=T_{2}=T_{w}=1.25$. After the equilibration stage, the upper wall moves with a $x$-velocity $u_{0}=1$. For the thermal problem, both walls are fixed. The initial temperature is uniform and set to $T_{1}=T_{2}=1$ before the upper wall temperature being increased to $T_{2}=1.5$.

The domain is simulated over the full height $H$ without applying any symmetry condition. The height of the molecular fluid region $\left(z \in\left[0 ; H_{M, f} / H\right]\right)$ and the continuum domains are identical, $H_{M, f}=H_{C}=139.21$, what leads to 
$H=H_{C}+H_{M, f}-H_{O}=222.74$ with an overlap region $H_{O}=40 \% H_{M, f}$. The transverse dimensions of the molecular block are $L_{x} \times L_{y}=9.8763 \times 9.775$. The molecular region contains $N=672$ atoms of argon in gas phase, such as the density $\rho=0.05$ is constant. The macroscopic coefficients evaluated at $T=1.25$ are $\mu=0.15, \lambda=0.62$ and $c_{p}=1.63$.

The ratio between the continuum and molecular time steps is $q=\Delta t / \delta t=400$. The microscopic variables stemming from the molecular dynamics are averaged in 10 horizontal slices of height $H_{M, f} / 10$, during one macroscopic time step $\Delta t=q \delta t$ and for 64 independent initial microscopic states also called samples.

To limit the velocity and temperature jumps occurring at the fluid-wall interface, the atomic wall is substituted by a stochastic wall model: when a particle crosses the plane at $z=0$ (Fig. 1), it is re-inserted with a velocity whose the tangential and normal components are sampled from a Gaussian and Rayleigh distributions respectively, with zero mean values and standard deviations corresponding to the wall temperature $T_{w}$ (Tehver et al., 1998; Xu et al., 2004). At the top of the molecular region, atoms leaving the domain are reflecting in a specular way by a fictive wall. An additional force, particularly useful for liquids, is applied to reduce the fluid stratification at the vicinity of this upper border (Werder et al., 2005) .

Figure 3 shows the numerical (symbols) and the analytical (lines) velocity and temperature profiles for the transient Couette and thermal problems as a function of the time. The good connection between the mean molecular and continuum profiles in the overlap region $(z / H \in[0.375 ; 0.56])$ and also the good agreement between the hybrid profiles and the analytical solutions validate the coupling scheme for the velocity and the temperature. Note that the error bars can be reduced with the increasing of the number of independent samples.

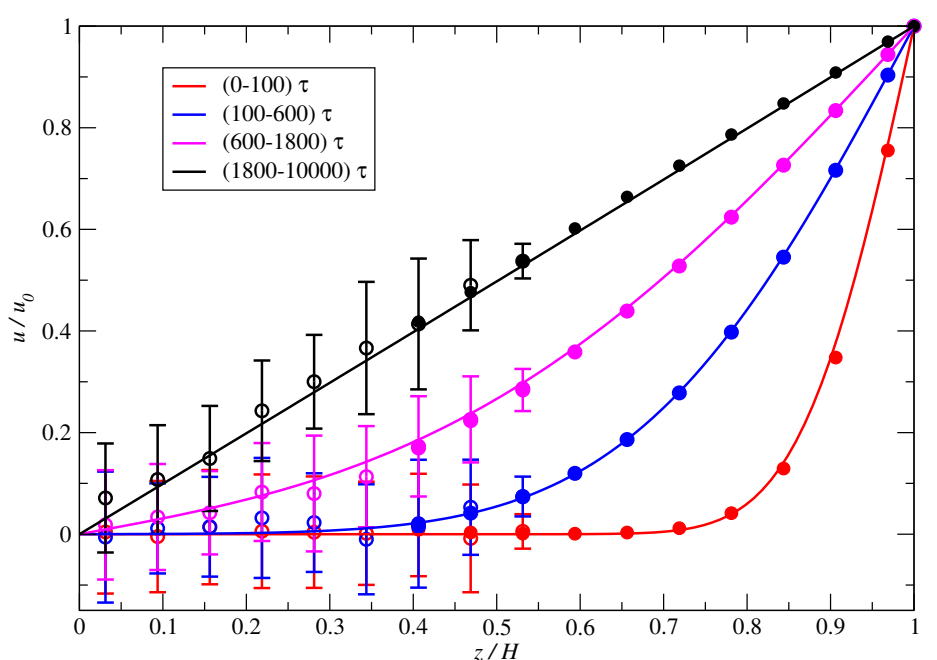

(a)

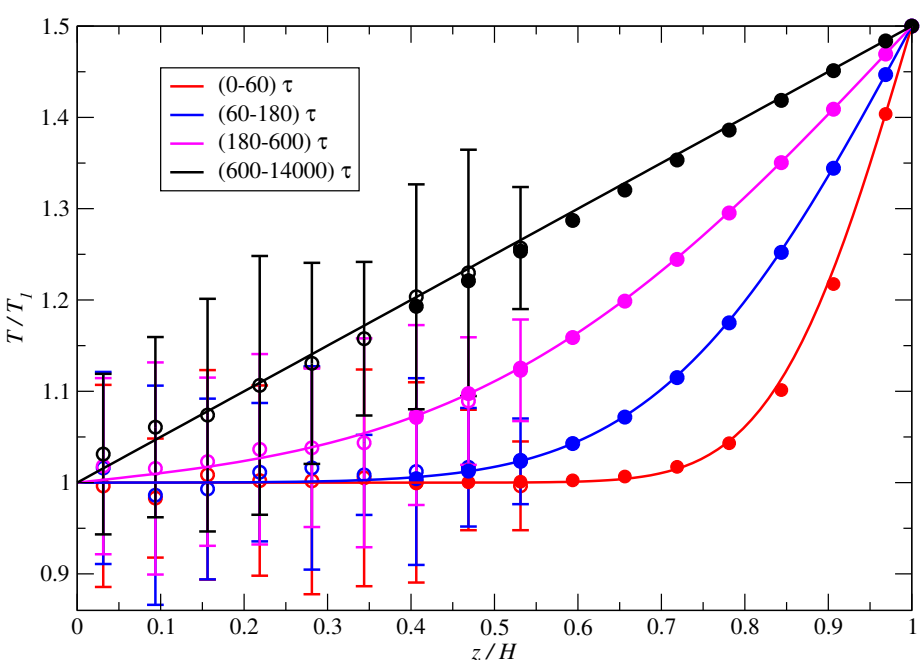

(b)

Figure 3: (a): Mean velocity $u / u_{0}$ and (b) temperature $T / T_{1}$ profiles for different time intervals. Continuous curves indicate the analytical solutions, filled and empty symbols stand for continuum and particle solutions of the hybrid method. The half lengths of the error bars represent the standard deviations and illustrate the scattering of the spatio-temporal averages over 64 independent samples.

\subsection{Control of the density}

The control method for the density is described in this section. The issue of the usefulness of a density control algorithm is first discussed via simple examples. Then, two variations of a main algorithm are presented and their relative efficiencies are studied on phase change problems.

\subsubsection{Discussion about the density control relevance}

The hybrid computations performed here concern academic problems. Let us consider a one-dimensional Poiseuille-like flow driven by a pressure gradient. The continuum part of the simulation is assumed incompressible with a fluid density $\rho=\rho_{\text {target }}$ and a viscosity $\mu=\mu\left(\rho_{\text {target }}, T_{\text {ref }}\right)$. In the $\mathrm{C} \rightarrow \mathrm{M}$ layer, the velocity and temperature are exchanged from the continuum to the molecular domain. When activated, the particle density $\langle\rho\rangle_{C \rightarrow M}$ must be driven to the targeted value $\rho_{\text {target }}$ which is, in our case, identical to the average initial density $\langle\rho\rangle_{\text {init }}$ of the molecular domain. The hybrid computations are performed for domain heights $H_{C} / H_{M, f}=2.45$, with $H_{M, f}=139.21$. The macroscopic coefficients are evaluated at the reference temperature $T_{\text {ref }}=1.25$ and density $\langle\rho\rangle_{\text {init }}=\rho_{\text {target }}=0.05$. The walls are modelled with 5 layers of platinum atoms arranged according to a FCC(111) lattice. The two most distant layers serve to keep the wall at rest and hold its temperature to 
$T_{w}=T_{\text {ref }}$ (Maruyama, 2000). The fluid flow is created by two values of the pressure gradient, $\Delta p / L_{x}=g_{0}$ and $4 g_{0}$, with the control of the molecular density $\langle\rho\rangle_{C \rightarrow M}$ or by keeping the density number to its initial value $\left(\langle\rho\rangle_{\text {init }}=\rho_{\text {target }}\right)$. The value of $g_{0}$ is $7.77 \times 10^{-7}$.

Figure 4 and Tab. 5 summarize the main results. When the pressure gradient is set to $g_{0}$, the viscous heating is nearly negligible. It results that the density in the molecular region is expected not to vary noticeably with respect to its initial value. Thus, applying or not a control of the density in the $\mathrm{C} \rightarrow \mathrm{M}$ layer should be neutral. This analysis is confirmed by the drawings of the profiles and the calculations of the mean and local values $\langle\rho\rangle$ and $\langle\rho\rangle_{C \rightarrow M}$, with a management of the density or a fixed number of fluid particles. Indeed, in each cases, the density values in the control layer depart of about $0.5 \%$ from the target value $\rho_{\text {target }}$ (Tab. 5). These differences are negligible since they are of the same order as the uncertainties stemming from the Molecular Dynamics computation and the numerical evaluation of the mean quantities. Notice that the insignificant increase in $\langle\rho\rangle_{C \rightarrow M}$ leads to a slight growth of $1.2 \%$ of the total number of the particles in the molecular domain. Finally, as expected, applying an algorithm to locally control the density of the molecular region is clearly useless when the density in the molecular region remains nearly constant and uniform.

For larger pressure gradients which produce higher flow rates, the temperature variation due to the viscous heating entails a non constant density profile. In that case, keeping the number of particles constant in the whole molecular domain is no longer justified. As illustrated in Fig. 4 for a pressure gradient $4 g_{0}$, the density profiles increase of about $30 \%$ by getting closer to the wall. A control method in the $\mathrm{C} \rightarrow \mathrm{M}$ layer is here essential to ensure the continuity with continuum region; in its absence, a jump of nearly $10 \%$ in the density values is observed (Tab. 5). Keeping $\langle\rho\rangle_{\mathrm{C} \rightarrow \mathrm{M}}$ to $\rho_{\text {target }}\left(=\langle\rho\rangle_{\text {init }}\right)$ implies to introduce or extract particles from the molecular domain. In our example, the number of molecules has been substantially increased by $11 \%$ with respect to the initial value $\langle\rho\rangle_{\text {init }}$.

In this section, we have highlighted the importance of the control in problems where the density profiles evolve noticeably, but smoothly. Other situations may lead to manage the continuity of the density variable, for example when the stratification of the fluid near the wall is significant or if a phase change occurs in the vicinity of the solid surface. The condensation and evaporation phenomena are exemplified in the next section.

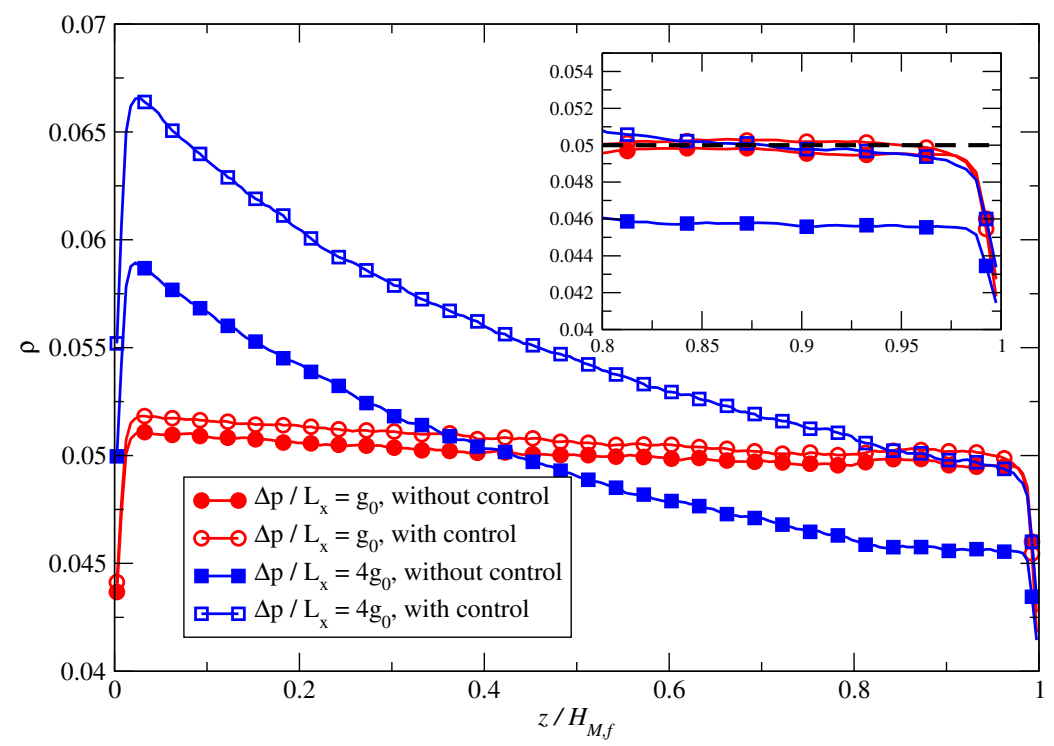

Figure 4: Density profiles in the molecular region of a Poiseuille-like flow subjected to two pressure gradients $\Delta p / L_{x}=g_{0}$ and $4 g_{0}$, with (open symbols) or without (filled symbols) the control of the density in the $\mathrm{C} \rightarrow \mathrm{M}$ layer. The encapsulated figure is an enlargement of the upper part of the overlap region. Numerical values are written out in Tab. 5 .

\subsubsection{Algorithm and numerical studies}

In the algorithm developed herein below, we assume that the density in the molecular domain is controlled by the continuum region, without feedback. This assumption is justified, for example in micro or nanochannel flows where the density field results mainly from the inlet and outlet boundary conditions which are applied to create the motion at large scale.

Let's recall that transfer of information from the continuum to molecular domain takes place in the $\mathrm{C} \rightarrow \mathrm{M}$ layer. Because the control of the density requires to modify the number of particles, it is then essential that the insertion and removal of molecules preserves the average velocity and the temperature (velocity standard deviation) stemming from the continuum. 


\begin{tabular}{|r||c|c||c|c|}
\hline \multicolumn{1}{|c||}{ Pressure gradient $\Delta p / L_{x}$} & \multicolumn{2}{c||}{$7.77 \times 10^{-7}$} & \multicolumn{2}{c|}{$31.1 \times 10^{-7}$} \\
\hline Control & without & with & without & with \\
\hline$\langle\rho\rangle$ & $\rho_{\text {init }}$ & 0.0506 & $\rho_{\text {init }}$ & 0.0554 \\
$\left(\langle\rho\rangle-\rho_{\text {init }}\right) / \rho_{\text {init }}$ & $0 \%$ & $1.2 \%$ & $0 \%$ & $11 \%$ \\
$\langle\rho\rangle_{C \rightarrow M}$ & 0.0497 & 0.0502 & 0.0457 & 0.0502 \\
$\left(\langle\rho\rangle_{C \rightarrow M}-\rho_{\text {target }}\right) / \rho_{\text {target }}$ & $-0.5 \%$ & $0.4 \%$ & $-8.5 \%$ & $0.4 \%$ \\
\hline
\end{tabular}

Table 5: Average densities over the entire molecular region, $\langle\rho\rangle$, and the $\mathrm{C} \rightarrow \mathrm{M}$ layer, $\langle\rho\rangle_{C \rightarrow M}$, with or without the control of the density. The deviations with respect to the initial and targeted densities are also written down $\left(\rho_{\text {init }}=\rho_{\text {target }}=0.05\right)$. Refer to Fig. 4 to look at the density profiles in the molecular region.

However, this condition is difficult to fulfil when using the constraint dynamics (Eq. (5)) with the Langevin thermostat (Eq. (6)), since any additional or removed molecule may disturb substantially the forces acting on particles. The approach taken in this work is to manage the density number, not in the $\mathrm{C} \rightarrow \mathrm{M}$ layer, but slightly above, in the control layer (Fig. 2(b)). The general algorithm is finally divided into two main steps: a local evaluation of the average density over $n$ molecular time iterations, followed by an instantaneous insertion or removal of particles in the control layer. This can be expressed as follows:

1. the averaged density $\langle\rho\rangle_{\mathcal{V}, n}$ in a sub-zone $\mathcal{V}$ of the molecular region is computed over the time interval $[t-n \times \delta t ; t$;

2. at time $t$, the difference between $\langle\rho\rangle_{\mathcal{V}, n}$ and the targeted density $\rho_{\text {target }}$ is converted into a number of atoms $|\Delta N|$ such that $\Delta N=\operatorname{int}\left[\left(\rho_{\text {target }}-\langle\rho\rangle_{\mathcal{V}, n}\right) \mathcal{V}\right]$, where int $(x)$ returns the nearest integer of the real value $x$. Depending on the algebraic value $\Delta N,|\Delta N|$ atoms are inserted $(\Delta N>0)$ or removed $(\Delta N<0)$ in the control layer.

The calculation of $\langle\rho\rangle_{\mathcal{V}, n}$ is straightforward: averages over the space $\mathcal{V}$, the number of time iterations $n$ and the number of samples are used ( Vu et al., 2016). Two sub-zones $\mathcal{V}$ have been tested to evaluate the density: the $\mathrm{C} \rightarrow \mathrm{M}$ layer, where the values of the horizontal velocity and temperature are passed from the continuum to molecular domain, and the control layer (Fig. 2(b)). The insertion of atoms $(\Delta N>0)$ rests on the USHER algorithm (Delgado-Buscalioni and Coveney, 2003): to minimize the disturbances, each additional atom is randomly put into the control layer and then driven at a position where its potential energy is equal to a specified target. For dilute gases, the target value is set to 0 , such that an atom is inserted in a zone without any interaction (the nearest atom is further away than the cut-off radius $r_{c}$ ). In that particular case, the USHER algorithm can be considered as a random insertion method since the descent step is seldom used. If $\Delta N<0$, the atoms with the smallest distances to the upper border are simply removed. Another simple molecule insertion process is proposed by Sun et al. (2011). The molecules are simply inserted at the centre of a tetrahedron formed by one molecule and its three nearest neighbours. Notice that the USHER algorithm could be substituted by the FADE algorithm (Borg et al., 2014) when the fluid consists of complex molecules (polymer chains for example).

The behaviour of the coupling algorithm is studied both on condensation and evaporation problems, with argon in gaseous or liquid phase. The wall temperature is chosen such that the phase change occurs in the molecular domain only. Then, the characteristics of the liquid/gas interface are determined by applying a nonlinear least-square fitting (Marquardt-Levenberg algorithm) on the density profile with the function

$$
\rho(\eta)=a \times \tanh \left(\frac{\eta-b}{c}\right)+d
$$

where $2|a|$ represents the jump of the density, $b$ is the interface middle ordinate, $2 c>0$ is a measure of its thickness and $d=\rho(b)$ is the mean density between the phases (Fig. 5 for a graphical interpretation of these coefficients).

The simulation parameters for the condensation problem are detailed as follows. The height of the molecular domain is $H_{M, f}=139.21$. The simulations are initialized with a density of gaseous argon $\rho_{\text {init }}=\rho_{\text {target }}=0.05$ and a temperature equal to $T_{\text {init }}=T_{w}=1.1$. The pressure gradient is set to $\partial_{x} p=-3.11 \times 10^{-6}$, and the horizontal velocity and the temperature in the $\mathrm{C} \rightarrow \mathrm{M}$ layer are kept at $u_{C \rightarrow M}=0.8$ and $T_{C \rightarrow M}=1.12$. After the initialization process, the wall temperature is decreased to $T_{w}=0.925$ and the steady state is sought.

Figure 6 shows the density in the molecular region, for the removal/insertion algorithm applied every $n=1,10,100,1000$ and 10000 time iterations. When both the evaluation and the density control are performed in the control layer (Fig. 6(a)), the interface position moves significantly with the density update period. Although the gas density seems to turn out to be insensitive to the numerical parameter $n$, a thorough examination of its behaviour in the $\mathrm{C} \rightarrow \mathrm{M}$ layer $\left(z / H_{M, f} \in[0.8 ; 0.9]\right)$ shows that its profile is not truly uniform and different from the expected value $\rho_{\text {target }}$ (horizontal discontinuous line). Thus, this difficulty in controlling the density may affect the correct position of the liquid-gas interface. These issues can be explained from the well known spurious stratification of the density which develops near the upper virtual border of the control layer (see the enlargement in Fig. 6(a)). It results a significant gradient as well as a poor estimation of the density at $z / H_{M, f}=0.9$, the junction between the control layer and the $\mathrm{C} \rightarrow \mathrm{M}$ region. The variations of both the interface characteristics and the density 


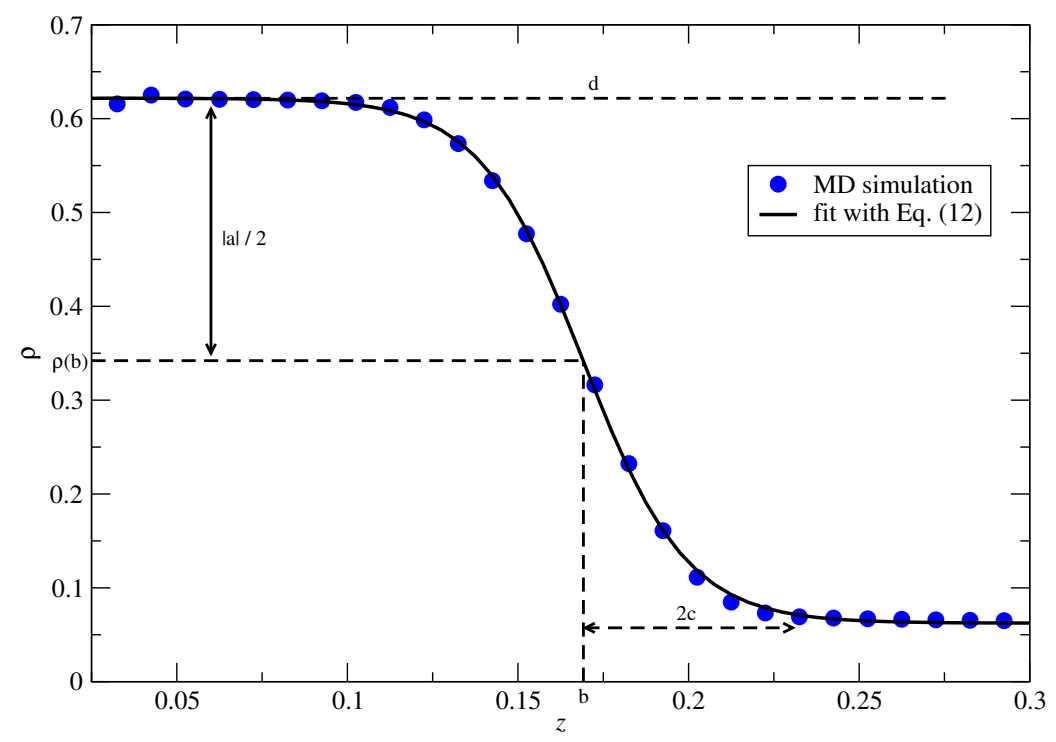

Figure 5: Density of the liquid/gas interface. The blue symbols represent the local molecular density and the continuous line indicates the fitted function. The meaning of the coefficients $a, b, c$ and $d$ of the approximation function Eq. (11) are also given.

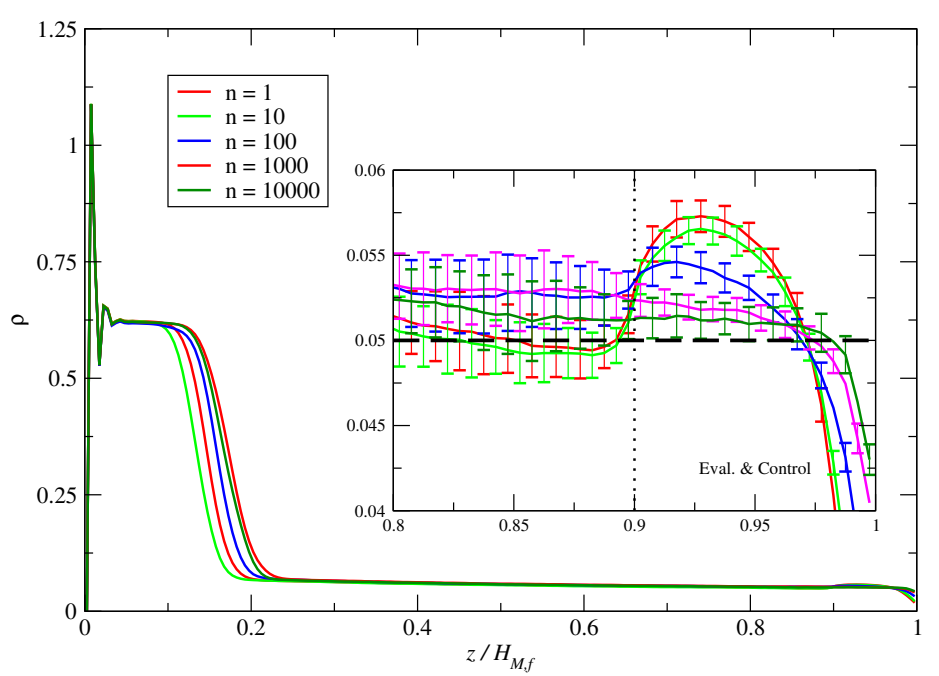

(a)

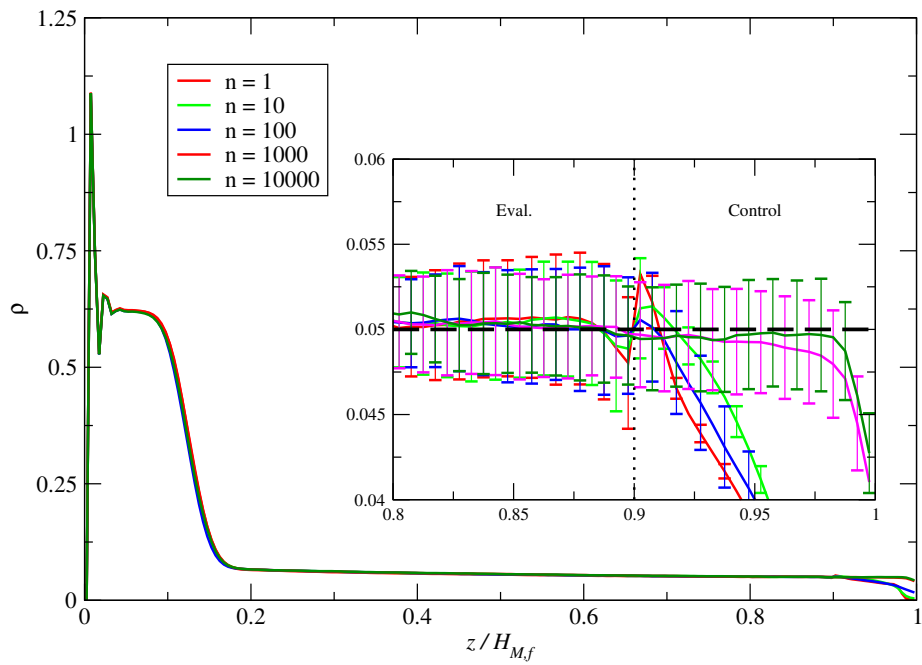

(b)

Figure 6: Condensation problem. Density profiles in the molecular region, for the removal/insertion algorithm applied every $n$ time iterations. The targeted density $\rho_{\text {target }}$ is indicated by the discontinuous line. (a): the evaluation and the control of the density are done in the control layer $\left(z / H_{M, f} \in[0.9 ; 1]\right)$. (b): the evaluation of the density takes place in the $\mathrm{C} \rightarrow \mathrm{M}$ layer $\left(z / H_{M, f} \in[0.8 ; 0.9]\right)$ whereas the density management is still performed in the control layer. The half error bars stand for the standard deviations calculated over 64 samples. Some quantities extracted from these curves are written out in Tab. 6.

$\langle\rho\rangle_{C \rightarrow M}$ as a function of $n$, the number of time iterations between two successive removal/insertion steps, are illustrated in Tab. 6 (column labelled One layer (control layer)). Even if the scattering of the density in the $\mathrm{C} \rightarrow \mathrm{M}$ layer is small $(\leq 1.4 \%)$, the relative difference between $\langle\rho\rangle_{C \rightarrow M}$ and $\rho_{\text {target }}$ ranges from $-0.4 \%$ to almost $6 \%$, with a non monotonous variation with $n$.

If the evaluation of the density is not done in the control layer, but in the $\mathrm{C} \rightarrow \mathrm{M}$ layer $(\mathcal{V}=\mathrm{C} \rightarrow \mathrm{M})$, the results look much more satisfactory (Fig. 6(b)). In that case, $\rho(z)$ is nearly uniform in $\mathcal{V}$ and well kept at $\rho_{\text {target }}$, whatever the value of $n$. These observations are confirmed by looking at the last set of columns Two layers (control and $C \rightarrow M$ layers) in Tab. 6. The location and thickness of the liquid/gas interface are quasi independent of the period $n$ of the molecule number update, and the density deviates from less than $0.7 \%$ from the target value.

It is worth noticing that, when the evaluation and the control of the density takes place in separated regions, the insertion or removal of particles do not affect immediately the value of the density $\langle\rho\rangle_{C \rightarrow M}$. Indeed, there exists a time delay $t_{D}$ which 


\begin{tabular}{|c||cc|ccc||cc|ccc|}
\hline \multicolumn{1}{|c||}{} & \multicolumn{4}{c||}{ One layer (control layer) } & \multicolumn{3}{c|}{ Two layers (control and C $\rightarrow$ M layers) } \\
\hline \multicolumn{1}{|c||}{ Interface } & $b$ & $c$ & $\langle\rho\rangle_{C \rightarrow M}$ & diff $\rho_{\text {target }}$ & $\frac{\sigma_{\langle\rho\rangle_{C \rightarrow M}}}{\langle\rho\rangle_{C \rightarrow M}}$ & $b$ & $c$ & \multicolumn{3}{c|}{ Density } \\
\hline$n$ & $b$ & 0.0240 & 0.05031 & $0.6 \%$ & $1.4 \%$ & 0.126 & 0.0264 & 0.05024 & $0.5 \%$ & $1.3 \%$ \\
\hline 1 & 0.147 & $0.024)$ & $\operatorname{diff}_{\rho_{\text {target }}}$ & $\frac{\sigma_{\langle\rho\rangle_{C \rightarrow M}}}{\langle\rho\rangle_{C \rightarrow M}}$ \\
10 & 0.135 & 0.0243 & 0.04978 & $-0.4 \%$ & $1.2 \%$ & 0.125 & 0.0269 & 0.05024 & $0.5 \%$ & $0.9 \%$ \\
100 & 0.159 & 0.0247 & 0.05270 & $5.4 \%$ & $0.4 \%$ & 0.123 & 0.0259 & 0.05023 & $0.5 \%$ & $0.6 \%$ \\
1000 & 0.173 & 0.0289 & 0.05293 & $5.9 \%$ & $0.4 \%$ & 0.126 & 0.0262 & 0.05025 & $0.5 \%$ & $0.4 \%$ \\
10000 & 0.168 & 0.0287 & 0.05165 & $3.3 \%$ & $0.9 \%$ & 0.125 & 0.0267 & 0.05034 & $0.7 \%$ & $0.7 \%$ \\
\hline$\langle\cdot\rangle$ & 0.156 & 0.0261 & 0.05147 & - & - & 0.125 & 0.0264 & 0.05026 & - & - \\
$\sigma \cdot /\langle\cdot\rangle$ & $10 \%$ & $9.5 \%$ & $2.7 \%$ & - & - & $1 \%$ & $1.5 \%$ & $0.1 \%$ & - & - \\
\hline
\end{tabular}

Table 6: Condensation problem with the evaluation of the density control applied in one or two different layers. Ordinate (b) and half thickness $(c)$ of the approximated interface $\rho(\eta)$ (Eq. (11) with a fitted interval $0.05 \leq \eta \leq 0.3$ ), averaged values of the density $\left(\langle\rho\rangle_{C \rightarrow M}\right)$ in the $\mathrm{C} \rightarrow \mathrm{M}$ layer, relative difference between the measured and the targeted density values $\left(\operatorname{diff}_{\rho_{\text {target }}}=\langle\rho\rangle_{C \rightarrow M} / \rho_{\text {target }}-1\right)$, and relative standard deviation of $\langle\rho\rangle(z)$ in the $\mathrm{C} \rightarrow \mathrm{M}$ layer $\left(\sigma_{\langle\rho\rangle_{C \rightarrow M}} /\langle\rho\rangle_{C \rightarrow M}\right)$, as a function of $n$, the number of time iterations between two successive removal/insertion steps. The two last lines give the averages and the relative standard deviations over all the $n$-values, for $b, c$ and $\langle\rho\rangle_{C \rightarrow M}$.

corresponds to the time necessary to diffuse the information from the control layer to the evaluation layer. This time scale is related to the self-diffusion coefficient $D$ and the length scale of the control layer, $\Delta z$. By assuming that the particles are inserted at an average distance $\Delta z / 2$ from the $\mathrm{C} \rightarrow \mathrm{M}$ layer, an approximation of this time delay is $t_{D}=(\Delta z)^{2} /(4 D)$. Knowing that $\Delta z=13.9$ and $D=2.5425 \sigma^{2} / \tau$ for a Lennard-Jones fluid at $\rho=0.055$ and $T=1$ (Meier, 2002), we obtain $t_{D} \simeq 19$ or $n_{D}=t_{D} / \delta t \simeq 3800$ molecular time iterations. If $\Delta \ll n_{D}$ (or $\Delta \times \delta t \ll t_{D}$ ), the inhomogeneities caused by the sudden change in the number of particles do not have enough time to diffuse, what creates abrupt changes in the density profile as observed in Fig. 6(b). Despite these disrupted profiles in the control layer, the averaged density $\langle\rho\rangle_{C \rightarrow M}$ remains very close to $\rho_{\text {target }}$, with negligible relative fluctuations $\sigma_{\langle\rho\rangle_{C \rightarrow M}} /\langle\rho\rangle_{C \rightarrow M} \leq 1.3 \%$.

Similar studies have been carried out for an evaporation problem. The molecular domain of height $H_{M, f}=49.72$ is initialized with liquid argon at $\rho_{\text {init }}=\rho_{\text {target }}=0.6$ and a temperature $T_{\text {init }}=T_{w}=1$. The pressure gradient is uniform, $\partial_{x} p=-9.86 \times 10^{-5}$, and the horizontal velocity and temperature in the $\mathrm{C} \rightarrow \mathrm{M}$ layer are controlled to $u_{C \rightarrow M}=0.53$ and $T_{C \rightarrow M}=T_{\text {init }}$. Then, the temperature of the platinum atoms is suddenly raised to evaporate the liquid in the vicinity of the wall. According to the phase diagram of Lennard-Jones fluids (Kolafa and Nezbeda, 1994), the wall temperature is set to $T_{w}=1.5 T_{C \rightarrow M}$. However, the numerical simulations show that this temperature value does not produce the expected evaporation due to the thermal resistance at the argon/platinum interface. To circumvent this issue, the wall temperature could be heightened. In this work, we choose to minimize the interface effect by increasing the well-depth of the potential between the argon and platinum to $\varepsilon_{f w}=6 \varepsilon$.

The density profiles in the molecular region are presented in Fig. 7 for several $n$-values corresponding to different time periods of insertion/removal of particles in the control layer. The results presented in Fig. 7(a) (resp. Fig. 7(b)) are obtained for an evaluation of the density in the control layer (resp. $\mathrm{C} \rightarrow \mathrm{M}$ layer). Table 7 indicates, for each of the plotted profiles, the ordinate and half-thickness of the approximated liquid/gas interface, the averaged value of the density in the $\mathrm{C} \rightarrow \mathrm{M}$ layer, its relative difference with the targeted density value and the spatial scattering of the density in the $\mathrm{C} \rightarrow \mathrm{M}$ layer. On the

\begin{tabular}{|c|c|c|c|c|c|c|c|c|c|c|}
\hline & \multicolumn{5}{|c|}{ One layer (control layer) } & \multicolumn{5}{|c|}{ Two layers (control and $\mathrm{C} \rightarrow \mathrm{M}$ layers) } \\
\hline & \multicolumn{2}{|c|}{ Interface } & \multicolumn{3}{|c|}{ Density } & \multicolumn{2}{|c|}{ Interface } & \multicolumn{3}{|c|}{ Density } \\
\hline$n$ & $b$ & $c$ & $\langle\rho\rangle_{C \rightarrow M}$ & $\operatorname{diff}_{\rho_{\text {target }}}$ & $\frac{\sigma\langle\rho\rangle_{C \rightarrow M}}{\langle\rho\rangle_{C \rightarrow M}}$ & $b$ & $c$ & $\langle\rho\rangle_{C \rightarrow M}$ & $\operatorname{diff}_{\rho_{\text {target }}}$ & $\frac{\sigma\langle\rho\rangle_{C \rightarrow M}}{\langle\rho\rangle_{C \rightarrow M}}$ \\
\hline 1 & 0.328 & -0.424 & 0.674 & $12 \%$ & $1.4 \%$ & 0.447 & -0.197 & 0.598 & $-0.3 \%$ & $3.9 \%$ \\
\hline 10 & 0.264 & -0.425 & 0.628 & $4.4 \%$ & $0.5 \%$ & 0.434 & -0.195 & 0.599 & $-0.2 \%$ & $1.7 \%$ \\
\hline 100 & 0.422 & -0.197 & 0.603 & $0.7 \%$ & $0.5 \%$ & 0.444 & -0.175 & 0.599 & $-0.2 \%$ & $0.4 \%$ \\
\hline 1000 & 462 & -0.157 & 0.596 & $-0.5 \%$ & $0.5 \%$ & 0.448 & -0.170 & 0.599 & $-0.2 \%$ & $0.5 \%$ \\
\hline 10000 & 0.471 & -0.147 & 0.595 & $-0.8 \%$ & $0.5 \%$ & 0.446 & -0.167 & 0.599 & $-0.2 \%$ & $0.5 \%$ \\
\hline$\langle\cdot\rangle$ & 0.389 & -0.270 & 0.619 & $\begin{array}{ll}- \\
-\end{array}$ & - & 0.435 & -0.181 & 0.599 & $\begin{array}{ll}- \\
-\end{array}$ & 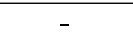 \\
\hline$\sigma \cdot /\langle\cdot\rangle$ & $23 \%$ & $53 \%$ & $5 \%$ & - & - & $1 \%$ & $8 \%$ & $0.1 \%$ & - & - \\
\hline
\end{tabular}

Table 7: Evaporation problem. Same caption as Tab. 6 but with a fitted interval for the function $\rho(\eta)$ (Eq. 11) equal to $0.1<\eta<0.9$.

whole, as in the condensation problem, the evaluation of the average density in the $\mathrm{C} \rightarrow \mathrm{M}$ layer gives more robust results, i.e. results very few dependent on $n$-values. However, some additional comments can be made. When the algorithm for the control of density is applied on a single layer, the liquid/gas interface may disappear (Fig. 7(a) for $n=1$ ). By looking 


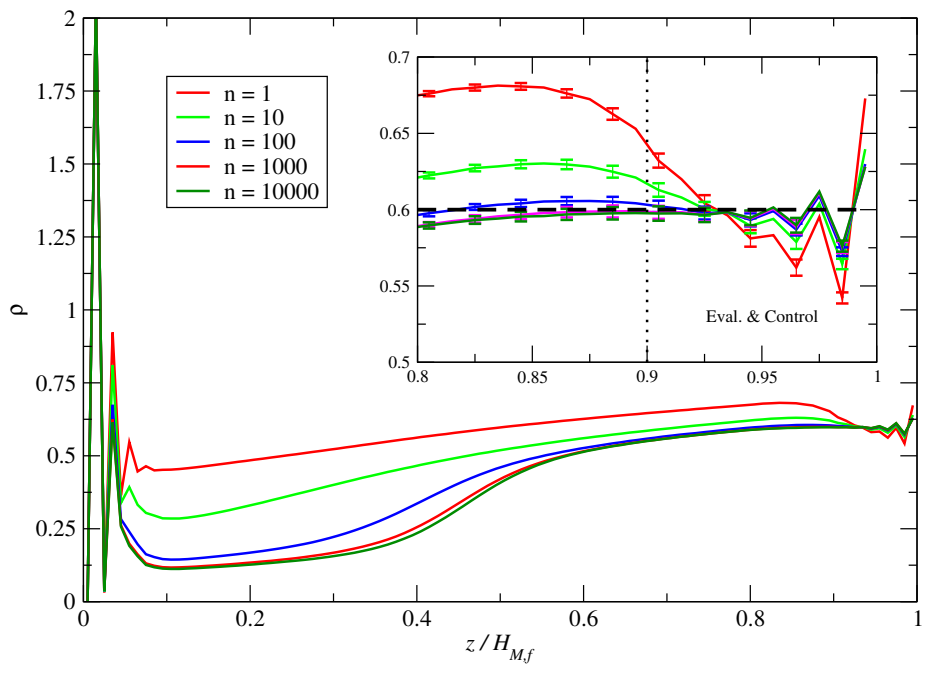

(a)

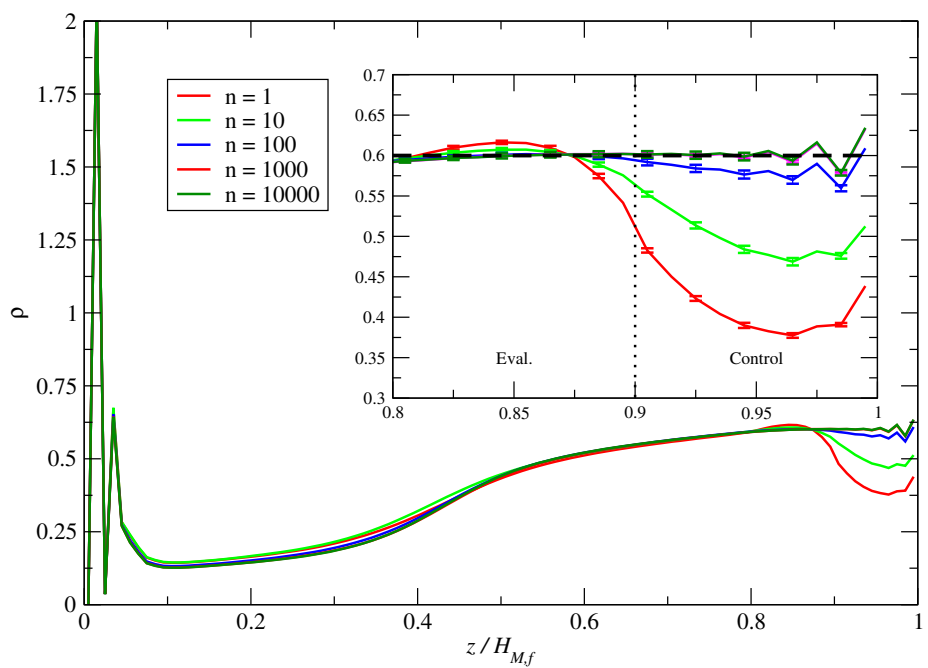

(b)

Figure 7: Evaporation problem. Same caption as Fig. 6. The half error bars stand for the standard deviations calculated over 32 samples. Some quantities extracted from these curves are written out in Tab. 7.

carefully at the encapsulated figure $7(\mathrm{~b})$, we see that the density profile remains smooth between the evaluation $\mathrm{C} \rightarrow \mathrm{M}$ layer $\left(0.8<z / H_{M, f}<0.9\right)$ and the control layer $\left(0.9<z / H_{M, f}<1\right)$ for $n \gtrsim 1000$. This lower limit is slightly smaller than $n_{D}=7200$, the approximation of the time iteration delay necessary to diffuse the sudden variation of the density number from the control to the evaluation layers. This order of magnitude is confirmed from the examination of Tab. 7 in which the interface thickness $c$ obtained for $n=10000$ departs of $4.8 \%$ from the solution computed with $n=100$, and then of $1.8 \%$ only when $n=1000$.

To conclude this section, only the algorithm with two separated layers for the evaluation of the molecular density and its control is really efficient to drive the density in the $\mathrm{C} \rightarrow \mathrm{M}$ layer. The obtained solutions are few sensitive to the period $n$ between two successive density number update. However, a good indicative value of the lower bound for $n$ may be given by the number of time molecular iterations based on the fluid self-diffusion coefficient and the control layer half length.

\subsection{Hybrid multiscale simulations}

In this section, we return to our main problem involving fluid flow and heat transfer in long narrow channels. The numerical simulations are carried out by the hybrid multiscale approach which presents the advantage of modelling both the small scales at the vicinity of the wall surface and the large scales generated by the pressure difference between the inlet and outlet sections.

To this purpose, several molecular blocks are distributed all along the fluid/wall interface. Since their number and positions affect significantly both the CPU time and the accuracy of the solutions, preliminary works must be conducted to optimize, at a lower cost, the distribution of these molecular blocks. As presented in Vu et al. (2016), the Molecular Dynamics simulations are substituted by analytical functions which define the temperature, velocity and density variables. These analytical expressions rest on approximations of the heat transfer and fluid flow which must be adapted to the physics studied. They must also assure the continuity with the continuum region, namely the analytical function and the discrete solution stemming from the continuum region must be equal at the centre of the $\mathrm{C} \rightarrow \mathrm{M}$ layer. It is worth pointing out that the analytical functions may also be used to cleverly initialize the Molecular Dynamics in order to speed up the convergence.

Two configurations are studied. The first one is a compressible gas flow where the density results mainly from the pressure drop, but also from the induced temperature field. The second problem is related to a gas flow with condensation at the channel walls. In each of these configurations, the number and the position of the blocks will be first defined by mean of the appropriate analytical functions, and then the hybrid multiscale simulations will be carried out.

\subsubsection{Compressible flow}

A compressible fluid flow into a long narrow channel of height $H=203.42$ and length $L=200 H$ is considered. The temperature at walls is set to $T_{w}=1.5$. The inlet fluid is in gaseous phase at the same temperature as the wall $T_{i n}=T_{w}$, the inlet density is $\rho_{\text {in }}=0.3$ that leads to the pressure $p_{\text {in }}=0.22$. The inlet/outlet pressure ratio is chosen as $p_{\text {in }} / p_{\text {out }}=2$. The height of the molecular fluid domain is one third of the continuum region $\left(H_{C} / H_{M, f}=3\right)$. The continuum region is divided 
into $N_{x} \times N_{z}=1500 \times 30$ uniform rectangular cells. The Navier-Stokes and energy equations (7) are solved in the steady state, i.e. by cancelling out the temporal derivatives. For that specific problem, the cut-off radius in the molecular region is $r_{c}=4$.

The analytical approximations of the thermal and velocity profiles in the different blocks are quite simple to obtain because the density and the pressure gradient are nearly constant in each cross-section. Given these remarks and assuming a fully developed stationary flow with constant properties in each of the $M_{i}$ blocks, the velocity function, $u_{M_{i}}^{\text {anal }}(z)$, is a piece of a parabola arising from a no-slip Couette-Poiseuille flow, driven by the constant pressure gradient $\left.\partial_{x} p\right|_{C \rightarrow M}$ and velocity $u_{M_{i}}^{\text {anal }}\left(z_{C \rightarrow M_{i}}\right)=u_{C \rightarrow M_{i}}$, both quantities transferred from the continuum domain. The temperature field is a solution of Eq. (7c) with the same assumptions as previously. It is expressed as a fourth order polynomial, where the integration constants are defined using the wall temperature $\left(T_{M_{i}}^{\text {anal }}(0)=T_{w}\right)$ and the coupling with the continuum domain $\left(T_{M_{i}}^{\mathrm{anal}}\left(z_{C \rightarrow M_{i}}\right)=T_{C \rightarrow M_{i}}\right)$.

Figure 8 presents the relative difference between the solution computed with a specific block distribution and a reference

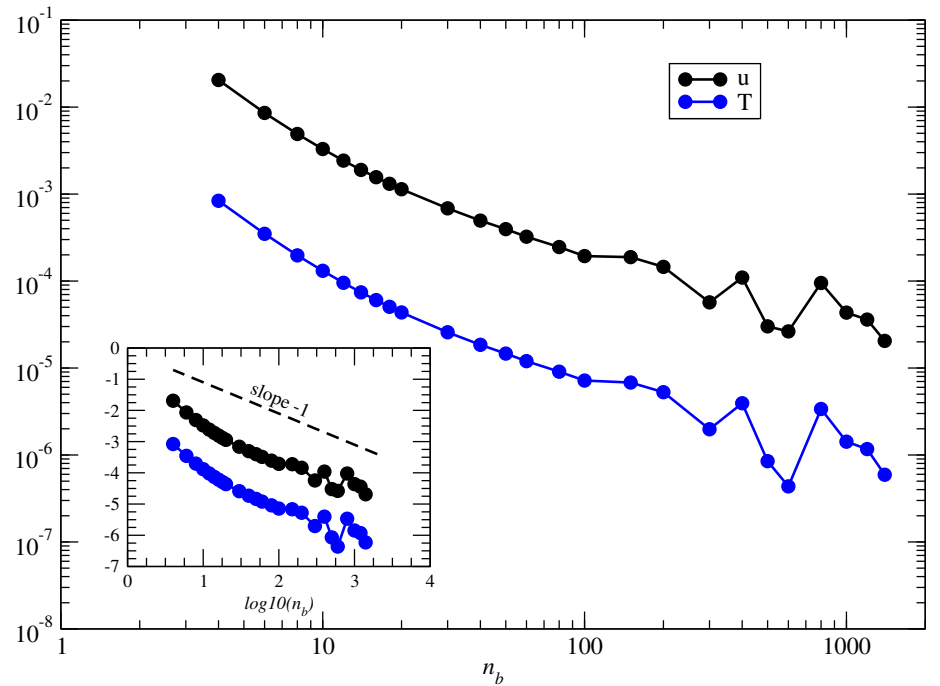

(a)

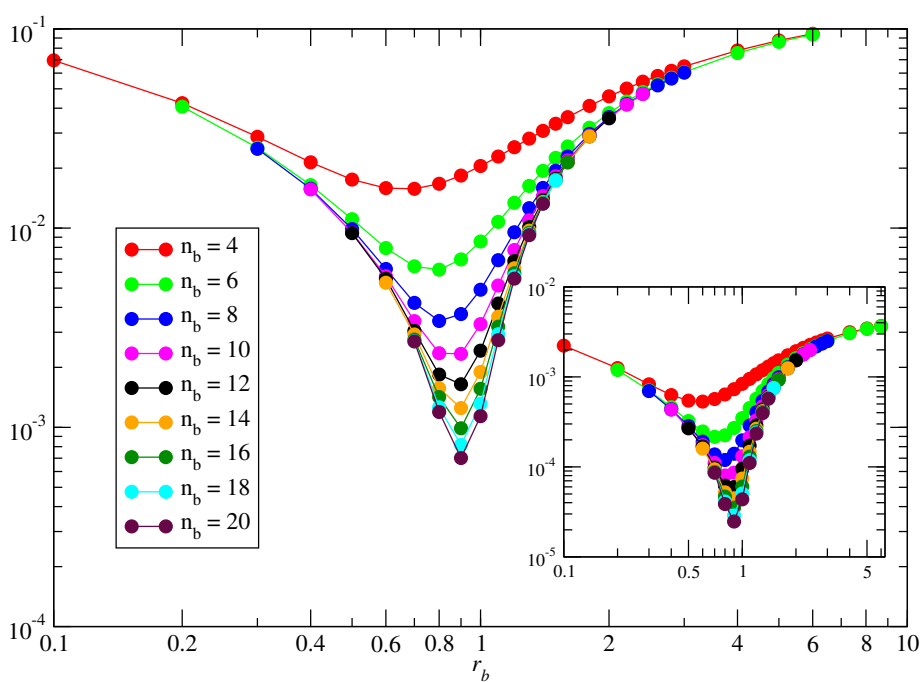

(b)

Figure 8: (a): Difference in $L_{2}$ norm of velocity and temperature fields computed by the analytical model for $n_{b}$ block and for $n_{b, \max }$ block. The encapsulated figure shows the $\log _{10}$ of the difference as a function of $\log _{10}\left(n_{b}\right)$ and suggest a first order convergence with the number of blocks. (b): Relative difference between the velocity field in the whole channel for a number of blocks $n_{b}$ positioned according to the scale factor $r_{b}$ and the reference solution with $n_{b}=N_{x}$. The encapsulated figure shows results for the temperature field.

solution carried out with just as many blocks as there are Finite Volume cells in $x$-direction $\left(n_{b, \max }=N_{x}\right)$. As shown in Fig. 8(a) for $n_{b}$ equi-spaced blocks, the multiscale simulation converges with $n_{b}$, on average at the first order. In order to reduce the number of blocks without decreasing the accuracy of the solution, a geometric progression with a scale factor $r_{b}$ $\left(r_{b} \neq 0\right.$ and 1$)$ is used to place them at the different abscissas $x_{i}$ :

$$
x_{i}=\frac{h_{1}}{2}+\left(L-\frac{h_{1}+h_{N_{x}}}{2}\right) \frac{1-r_{b}^{i-1}}{1-r_{b}^{n_{b}-1}}, \quad i=1, \ldots, n_{b}
$$

with $h_{1}$ and $h_{N_{x}}$ the sizes of the first and last cells in $x$-direction. As shown in Fig. 8(b), the best value for the scale factor is close to $r_{b}=1$, namely equi-spaced blocks. In the hybrid multiscale simulations performed hereinbelow we have chosen $n_{b}=8$ molecular blocks with $r_{b}=0.8$.

Figure 9 gives the velocity, temperature and density profiles in the $x_{i}$ cross-sections, for the hybrid multiscale solution and the Finite Volume approximation conducted on the whole fluid domain $[0 ; L] \times[0 ; H]$, with $H=H_{C}+H_{M, f}-H_{O}$ (Fig. 2 (b)). The length $H_{M, f}-H_{O}$ of the molecular fluid domain is substituted by a row of Finite Volume cells of same height. The continuity between the molecular (open circles) and continuous solutions (filled circles) in the overlap region is quite satisfying, except maybe for the density in the two sections closest to the channel entrance, $x_{1} / H \approx 0$ and $x_{2} / H \approx 50$ : the molecular densities evaluated in the $\mathrm{C} \rightarrow \mathrm{M}$ layer is $0.8 \%$ smaller than expected. This value is in the order of magnitude of the errors found when the algorithm for the control of the density has been tested (see column diff $\rho_{\text {target }}$ for two layers in Tab. 6). The comparison between the multiscale hybrid (symbols) and the Finite Volume results (continuous line) shows a good agreement. The small deviations observed in the temperature and velocity profiles are due to the thermal and velocity slips which are authorized 


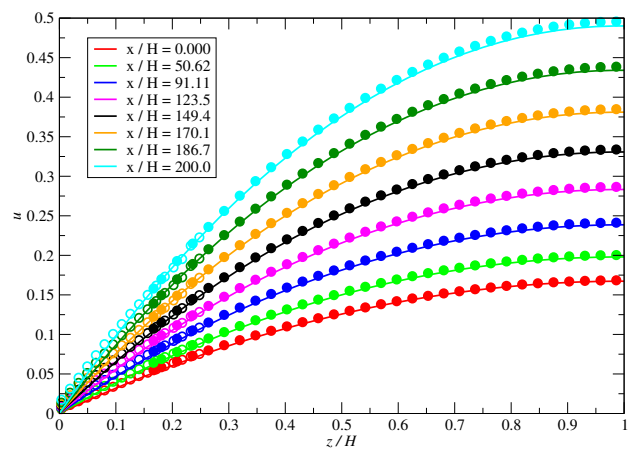

(a)

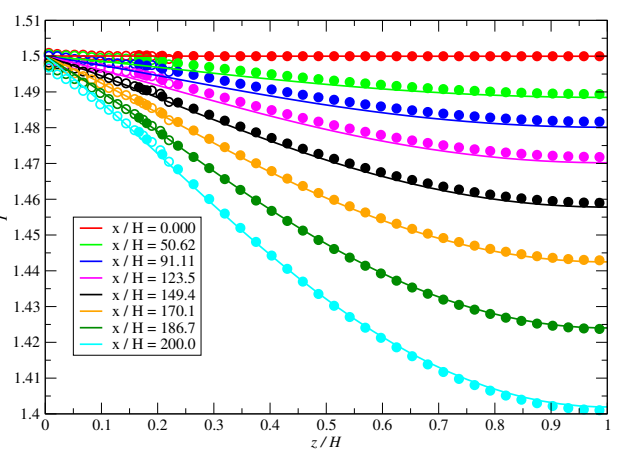

(b)

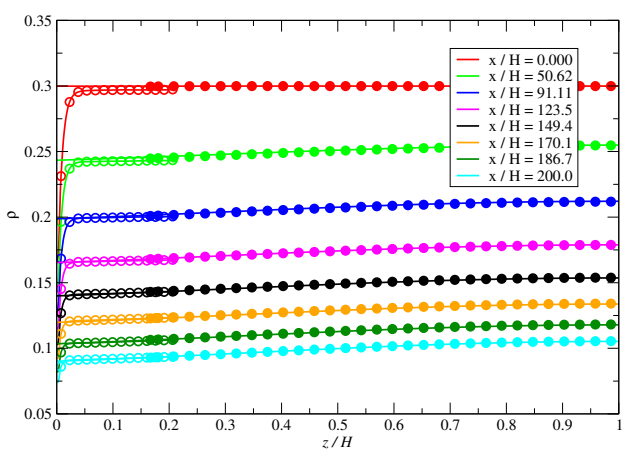

(c)

Figure 9: Profiles of the horizontal velocity $u$ (a), the temperature (b) and the density (c) at abscissas corresponding to the positions of the molecular blocks. Symbols show the hybrid multi scale simulation; empty and fill symbols plot the molecular and the continuum solutions, respectively; lines present the pure macroscopic solution without velocity and temperature slip at walls. Simulations are performed over 192 samples.

in the molecular simulation, only. Likewise, the sudden drop of the density near the wall is due to the stratification of the gaseous molecules in the vicinity of the solid platinum atoms.

The physical analysis of the multiscale hybrid solution indicates that the velocity slip (Fig. (9(a))) and temperature jump (Fig. (9(b))) are small, about $2.5 \%$ of the maximal velocity and maximal temperature difference. As expected for compressible flows, the fluid gets moderately colder by moving away from the inlet section, $-6.6 \%$ of $T_{w}$ (Fig. (9(b))). Indeed, two contributions of opposite signs intervene in the thermal balance (Eq. (7c)): $[\overline{\bar{\tau}}: \nabla \mathbf{u}]$ and $[-(\partial \ln \rho)(\partial \ln T) \mathbf{u} . \nabla p]$. Contrary to the viscous work which provides heat to the fluid, the pressure work removes some of it. In the case of microchannels, the cooling effect due to the pressure work is usually more intense than the viscous heating. It results a weak increase in the density profiles by getting closer to the core flow (Fig. $(9(\mathrm{c}))$ ). The significant drop in the density is caused by the imposed pressures, $p_{\text {in }}$ and $p_{\text {out }}$, in the inlet/outlet sections. By neglecting the effect of the temperature, the decrease in the density between the entrance and exit sections of the channel is much larger than the expected value $p_{\text {in }} / p_{\text {out }}=2$, which would be obtained for a gas law equation. The increase in the velocity along the channel (Fig. (9(a))) is proportional to the decrease in the density (mass flow rate conservation).

\subsubsection{Gas flow with condensation at the channel walls}

The condensation problem of gaseous argon flowing into a narrow channel of height $H=424.6$ and $L=500 H$ is now considered. The temperature at wall is set to $T_{w}=0.925$. The gas enters the channel with a density $\rho=0.05$, at the prescribed velocity $u_{i n}(z)$ and temperature $T_{i n}(z)$. These functions correspond to a fully developed solution of an incompressible fluid flow with a maximal velocity $u_{0}=2$ and a wall temperature $T_{w, 0}=1.1>T_{w}$. By considering the viscous heating, the velocity and temperature profiles in the inlet section read

$$
u_{i n}(z) / u_{0}=(z / H)(2-z / H), \quad T_{i n}(z)=T_{w, 0}+\mu u_{0}^{2}\left(1-(1-z / H)^{4}\right) / 3 \lambda
$$

The argon properties are evaluated at the temperature 1.25 , a value close to $T_{i n}(z=H / 2): \mu=0.15, \lambda=0.62$ and $c_{p}=1.63$. The characteristics of the hybrid simulation are $H_{M, f}=139.21$ and $H_{C} / H_{M, f}=2.45$. The continuum region is partitioned into a $N_{x} \times N_{z}=48000 \times 30$ uniform rectangular mesh. The Navier-Stokes and energy equations (7) are solved in their incompressible steady form, by disregarding all the time derivatives and the pressure work term in the energy equation (7c), and keeping the density constant.

The formulation of the analytical model devoted to approximate the heat transfer and fluid flow in the molecular block $M_{i}$ is based on the following assumptions: the solution is stationary and fully developed, the physical properties are constant for each phase and the pressure gradient is uniform in each block section $\left(\mathrm{d} p /\left.\mathrm{d} x\right|_{C \rightarrow M_{i}}\right)$. We also assume the continuity of the velocity and temperature at the liquid/gas interface. Contrary to the dynamical boundary condition where the fluid adheres to the wall, a thermal jump is taken into account at the solid interface. To distinguish the different phases of the fluid, the subscripts $l$ and $g$ are added to the liquid and gas quantities, respectively. By denoting the analytical thickness of the condensation film as $\delta^{\text {anal }}$, the Navier-Stokes and energy equations reduce to

$$
\text { for } 0 \leq z \leq \delta^{\text {anal }}:\left\{\begin{array}{l}
-\left.\frac{\mathrm{d} p}{\mathrm{~d} x}\right|_{C \rightarrow M_{i}}+\mu_{l} \frac{\mathrm{d}^{2} u_{l}^{\text {anal }}}{\mathrm{d} z^{2}}=0 \\
\lambda_{l} \frac{\mathrm{d}^{2} T_{l}^{\text {anal }}}{\mathrm{d} z^{2}}+\frac{\mu_{l}}{2} u_{l}^{\text {anal }} \frac{\mathrm{d} u_{l}^{\text {anal }}}{\mathrm{d} z}=0
\end{array} \quad, \quad \text { for } \delta^{\text {anal }} \leq z \leq z_{C \rightarrow M_{i}}:\left\{\begin{array}{l}
-\left.\frac{\mathrm{d} p}{\mathrm{~d} x}\right|_{C \rightarrow M_{i}}+\mu_{g} \frac{\mathrm{d}^{2} u_{g}^{\text {anal }}}{\mathrm{d} z^{2}}=0 \\
\lambda_{g} \frac{\mathrm{d}^{2} T_{g}^{\text {anal }}}{\mathrm{d} z^{2}}+\frac{\mu_{g}}{2} u_{g}^{\text {anal }} \frac{\mathrm{d} u_{g}^{\text {anal }}}{\mathrm{d} z}=0
\end{array}\right.\right.
$$


with the set of boundary conditions

$$
\text { at } z=0:\left\{\begin{array}{l}
u_{l}^{\text {anal }}=0 \\
T_{l}^{\text {anal }}-T_{w}=l_{s} \frac{\mathrm{d} T_{l}^{\text {anal }}}{\mathrm{d} z},
\end{array} \text { at } z=z_{C \rightarrow M_{i}}:\left\{\begin{array}{l}
u_{g}^{\text {anal }}=u_{C \rightarrow M_{i}} \\
T_{g}^{\text {anal }}=T_{C \rightarrow M_{i}}
\end{array}, \text { at } z=\delta^{\text {anal }}:\left\{\begin{array}{l}
u_{l}^{\text {anal }}=u_{g}^{\text {anal }} \\
T_{l}^{\text {anal }}=T_{g}^{\text {anal }}=T_{l / g} \\
\mu_{l} \frac{\mathrm{d} u_{l}^{\text {anal }}}{\mathrm{d} z}=\mu_{g} \frac{\mathrm{d} u_{g}^{\text {anal }}}{\mathrm{d} z} \\
\mathrm{~d} T_{l}^{\text {anal }}=\lambda_{g} \frac{\mathrm{d} T_{g}^{\text {anal }}}{\mathrm{d} z}
\end{array}\right.\right.\right.
$$

From this set of equations (14a) and boundary conditions (14b), it is possible to express the condensation thickness $\delta^{\text {anal }}$ as the solution of a non-linear equation $f_{\mathcal{X}}\left(\delta^{\text {anal }}\right)=0$, where $\mathcal{X}=\left\{\mu_{l}, \mu_{g}, \lambda_{l}, \lambda_{g}, z_{C \rightarrow M_{i}}, \mathrm{~d} p /\left.\mathrm{d} x\right|_{C \rightarrow M_{i}}, u_{C \rightarrow M_{i}}, T_{C \rightarrow M_{i}}, T_{w}, T_{l / g}, l_{s}\right\}$ is a set of parameters. The conductivities $\left(\lambda_{l}, \lambda_{g}\right)$ and viscosities $\left(\mu_{l}, \mu_{g}\right)$ are constant, the ordinate $z_{C \rightarrow M_{i}}$ is the centre of the layer $\mathrm{C} \rightarrow \mathrm{M}_{i}$, the pressure gradient $\mathrm{d} p /\left.\mathrm{d} x\right|_{C \rightarrow M_{i}}$, the velocity $u_{C \rightarrow M_{i}}$ and temperature $T_{C \rightarrow M_{i}}$ are passed from the continuum region, the wall temperature $T_{w}$ is fixed, and the phase change temperature $T_{l / g}$ and the thermal slip length $l_{s}$ (also known as the Kapitza length) have to be defined. The evaluation of $T_{l / g}$ deserves some comments. The saturation curve $T_{l / g}(p)$ can, a priori, be determined as soon as the equation of state is known. But, since the Lennard-Jones equation is a result of an analytical fitting of numerous numerical experiments, the temperature $T_{l / g}(p)$ suffers from some inaccuracies. Moreover, this temperature is defined at the thermodynamic equilibrium, a condition which is not really fulfilled by our condensation problems, because of the large temperature gradients created by the viscous heating. Lastly, the solution of the non-linear equation $f_{\mathcal{X}}\left(\delta^{\text {anal }}\right)=0$ turns out to be very sensitive to the condensation temperature: $+0.1 \%$ variation on $T_{l / g}$ increases $\delta^{\text {anal }}$ by $6.5 \%$. To overcome these issues, hybrid numerical simulations are carried out to calibrate $T_{l / g}$, as well as the thermal slip length $l_{s}$.

As an example, we present the density, velocity and temperature profiles obtained for the hybrid simulation with $\left.\partial_{x} p\right|_{C \rightarrow M}=$ $-3.11 \times 10^{-6}$ (Fig. 10). In the molecular region (empty symbols), the variations between the liquid and gas phase of the

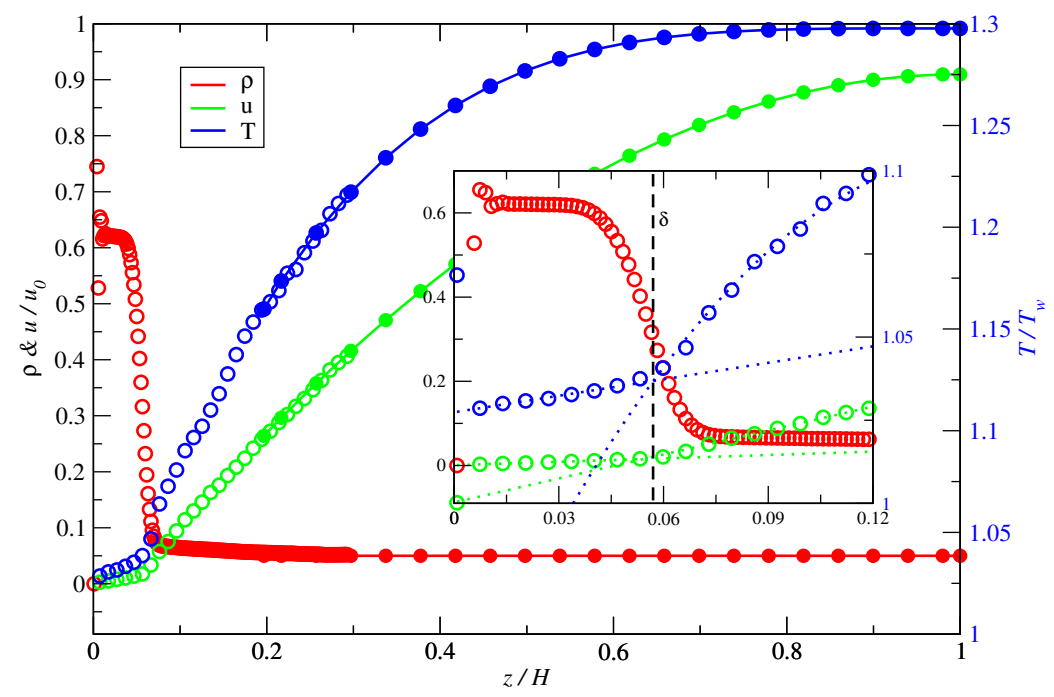

Figure 10: Profiles of density and horizontal velocity (left axis) and temperature (right axis) for and hybrid simulation on a single block with $\left.\partial_{x} p\right|_{C \rightarrow M}=-3.11 \times 10^{-6}$. Empty symbols stand for the molecular region while fill symbols and lines are the continuum solution. The encapsulated figure presents a zoom of the interface; linear or quadratic regression are performed over the velocity and temperature profiles to approach the interface position $\delta$.

transport coefficients $\mu$ and $\lambda$ involve sudden changes in the velocity and temperature slopes. These discontinuities in the derivatives arise around $z / H=0.056$, a numerical value which correspond to the mean thickness of the liquid film, $\delta=0.056 H$. Unlike the analytical model, the interface in the hybrid simulation is diffuse: the variation of the density is spread over approximately $3 \%$ of $z / H$, from $\rho_{l} \simeq 0.61$ to $\rho_{g} \simeq 0.05$. By following the same approach as in Sec. 3.2.2, the density profile fitted by an hyperbolic tangent function (Eq. (11)) in the range $z / H \in[0.05 ; 0.15]$ provides an interface thickness $1.8 \%$ smaller than previously, about $0.055 H$. Once $\delta$ is known, the phase change temperature reads $T_{l / g} \stackrel{\text { def }}{=} T(\delta)=1.04 T_{w}$. From the hybrid simulation, the thermal slip length $l_{s}$, defined by $l_{s} \stackrel{\text { def }}{=}\left[\left(T(z=0)-T_{w}\right) /\left.\partial_{z} T\right|_{z=0}\right]$, is approximated by $l_{s}=60.5$. By setting the liquid and gas properties to $\left(\mu_{l} ; \mu_{g}\right)=(0.82 ; 0.15),\left(\lambda_{l} ; \lambda_{g}\right)=(3.40 ; 0.62)$, and using the exchanged variables $u_{C \rightarrow M}=0.8$ and $T_{C \rightarrow M}=1.11$ for $z_{C \rightarrow M}=0.85 H_{M, f}$, the solution of a non-linear equation $f_{\mathcal{X}}\left(\delta^{\text {anal }}\right)=0$ writes $\delta^{\text {anal }}=0.05486 H$, namely $2 \%$ smaller than the measured quantity $\delta$. Based on 6 hybrid simulations for the pressure gradients $\left.\partial_{x} p\right|_{C \rightarrow M} \in-\{0.9 ; 1.0 ; 1.05 ; 1.1 ; 1.15 ; 1.2\} \times 3.11 \times 10^{-6}$, laws of variation for the thermal slip length and the phase change temperature can be developed: $l_{s} \approx 60.5$ and $T_{l / g} \approx 0.152 T_{C \rightarrow M}+0.786$. Once the whole parameters $\mathcal{X}$ of the analytical 
model (14) are known, the optimal block positioning can be carried out with the multiscale simulations.

Figure 11 illustrates the velocity and temperature relative differences committed between the multiscale models using $n_{b}$ and

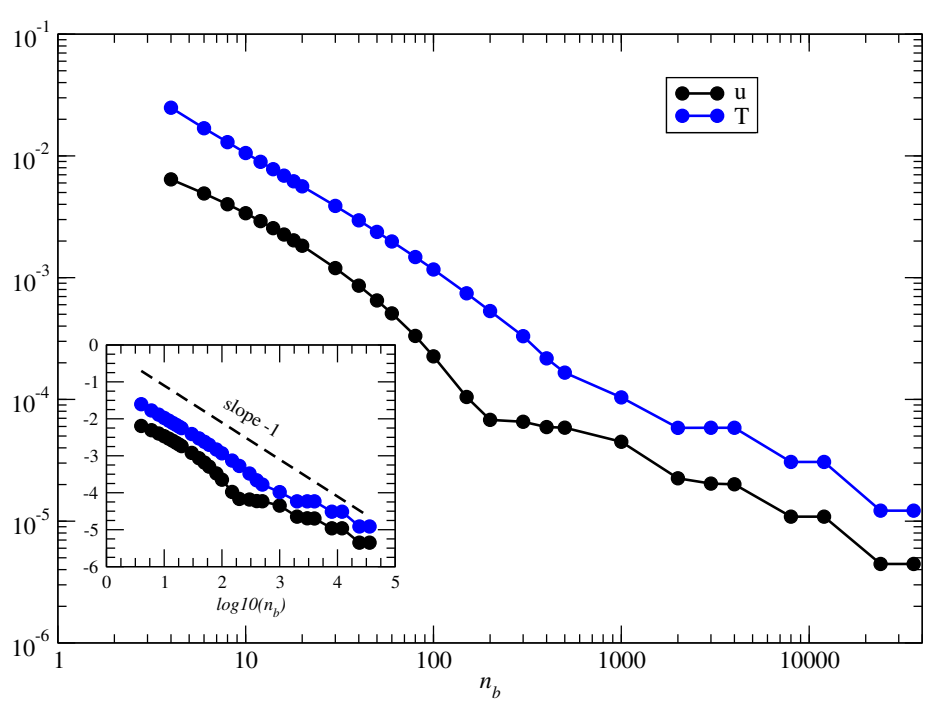

(a)

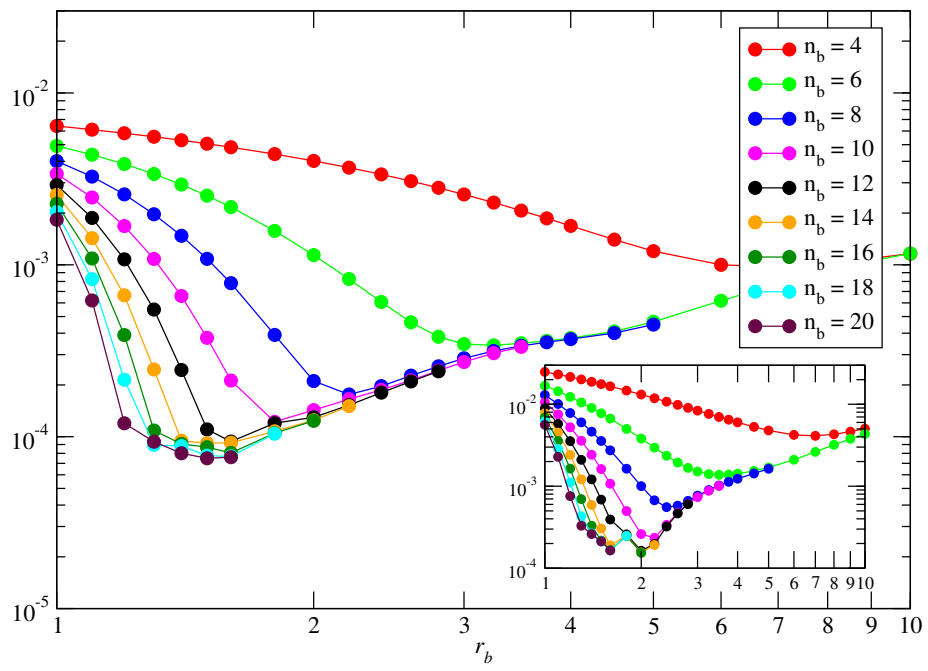

(b)

Figure 11: (a): Difference in $L_{2}$ norm of velocity and temperature fields computed by the analytical model for $n_{b}$ block and for $n_{b, \text { max }}$ block. The encapsulated figure shows the $\log _{10}$ of the difference as a function of $\log _{10}\left(n_{b}\right)$ and suggest a first order convergence with the number of blocks. (b): Relative difference between the velocity field in the inlet region $(x / H<300)$ for a number of blocks $n_{b}$ positioned according to the scale factor $r_{b}$ and the reference solution with $n_{b}=N_{x}$. The encapsulated figure shows results for the temperature field.

$n_{b, \max }=N_{x}$ analytical blocks, for an equi-spaced (left) and a non uniform (right) distributions of the analytical blocks. Contrary to the compressible flow example (Sec. 3.3.1), where a quasi uniform distribution of blocks was satisfactory, a scale factor $r_{b}$ greater than 1 is now preferable in Eq. (12). A good compromise between the accuracy and the computation cost is $n_{b}=10$ and $r_{b}=2$ : with that choice, the relative differences for the velocity and temperature do not exceed $0.1 \%$.

The velocity and temperature profiles in different cross-sections corresponding to the molecular block abscissas, for the hybrid multiscale simulation, are presented in Fig. 12. The hot gas enters into the channel equipped with cold walls, condenses and generates a thin liquid film along the fluid/solid interface. The appearance of this condensate modifies slightly the velocity field in the vicinity of the wall. As assumed in the analytical model, the fluid flow adheres to the wall. On the other hand, a significant thermal slip in the order of $20 \%$ to $30 \%$ of the temperature difference $T(z=H)-T(z=0)$ is observed. This temperature variation is due to the fluid cooling by the cold wall.

While the Molecular Dynamics and the Finite Volume solutions are generally very close in the overlap region (see the encapsulated graphs in Fig. 12), noticeable gaps are evident on the thermal profile drawn for the first abscissa $x_{1}$ (red symbols in Fig. 12(b)). Indeed, there is an inconsistency between the inlet temperature calculated at wall (see Eq. (13)) and the wall temperature. This problem translates into a condensate thickness visible in the first molecular block, while the continuous temperature remains very close to thermal field imposed at the entrance of the channel. This particular issue is not really surprising because the coupling between the molecular and continuum region is assumed unidimensional. In other words, the solution computed in the first molecular block is never affected by the inlet boundary conditions. To avoid that, the simulation in the first molecular block should be also coupled with the inlet solution (two dimensional coupling).

The condensate thicknesses obtained with the multiscale hybrid model and the analytical model are presented in Fig. 13 as a function of the block abscissas. Past a few channel heights, the result from the multiscale hybrid analytical model (black line) is in good agreement with that from atomistic-continuum coupling model (blue line). The condensate thickness computed with the analytical model (red line), in which the thermal slip length $l_{s}$ and the phase change temperature $T_{l / g}$ were extracted from each molecular block of the multiscale hybrid numerical simulations, is also shown. The agreement with the numerical simulation is excellent. This shows that the analytical model (14) is capable to represent accurately the flow and heat transfer occurring in the molecular blocks. It is surprising to observe that the condensate film thickness, calculated from the multiscale hybrid simulation, decreases as a function of $x / H$. Indeed, considering that the liquid wall temperature $T(x, z=0)$ decreases away from the inlet section (Fig. 12(b)), a thickening of the condensate would rather have been expected. To explain this counter intuitive behaviour, we must examine both the temperature jump $T(x, z=0)$ and the phase change temperature $T_{l / g}(x)$. 


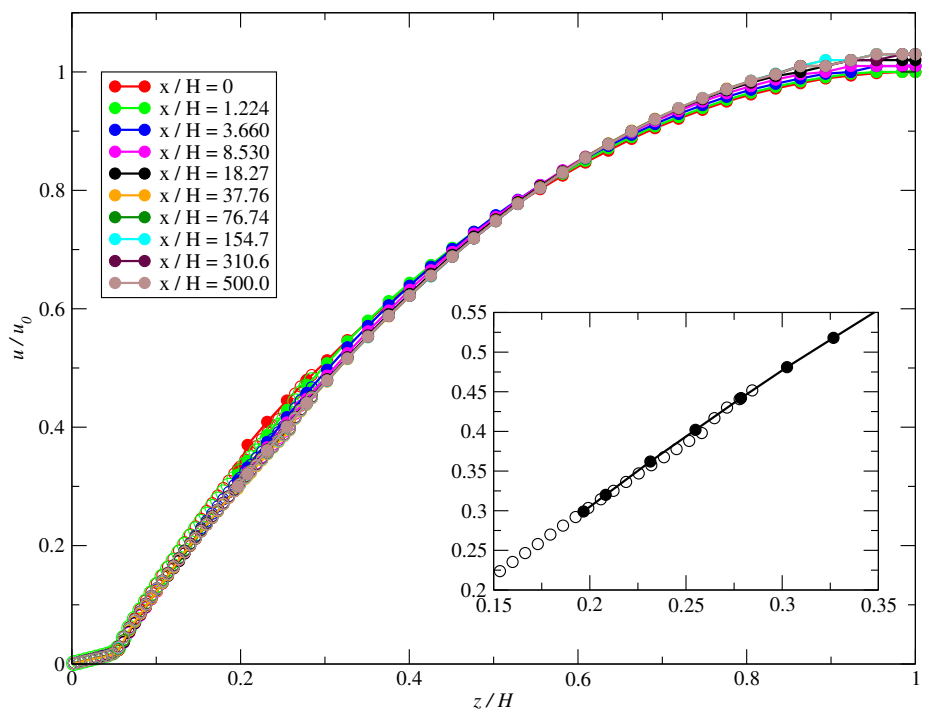

(a)

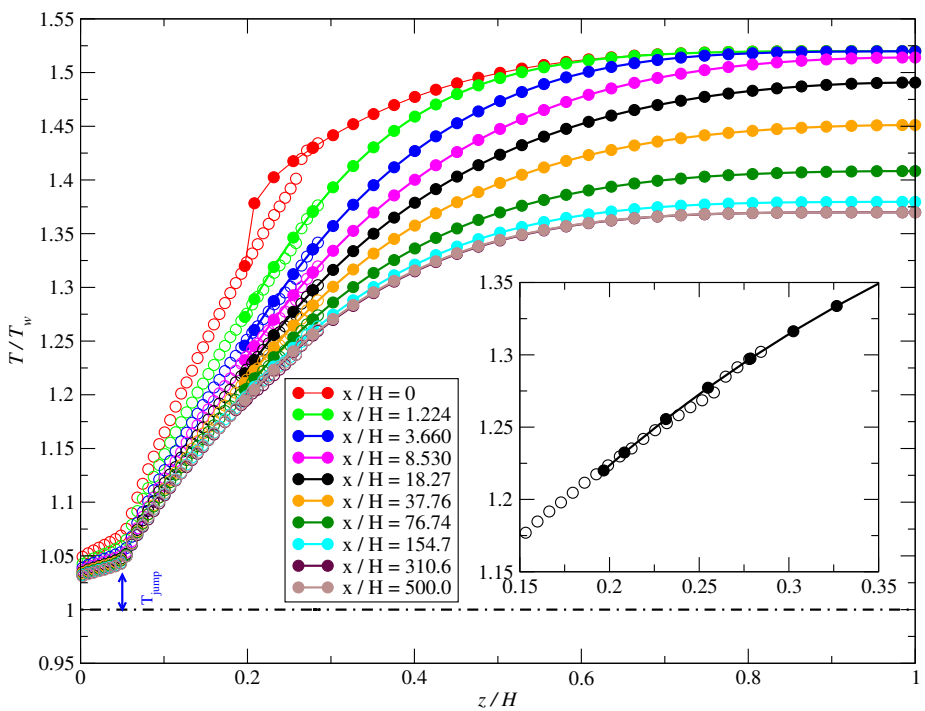

(b)

Figure 12: (a): Horizontal velocity $u / u_{0}$ profiles in slices corresponding to the molecular blocks positions in the channel. Generally, the velocity in the overlap region and the continuum model are in good agreement. A zoom of the overlap region is plotted in the encapsulated figure for the 5-th block to illustrate the coupling. (b): Same as Fig. 12(a) for the temperature field $T / T_{w}$. Simulations are performed over 192 samples.

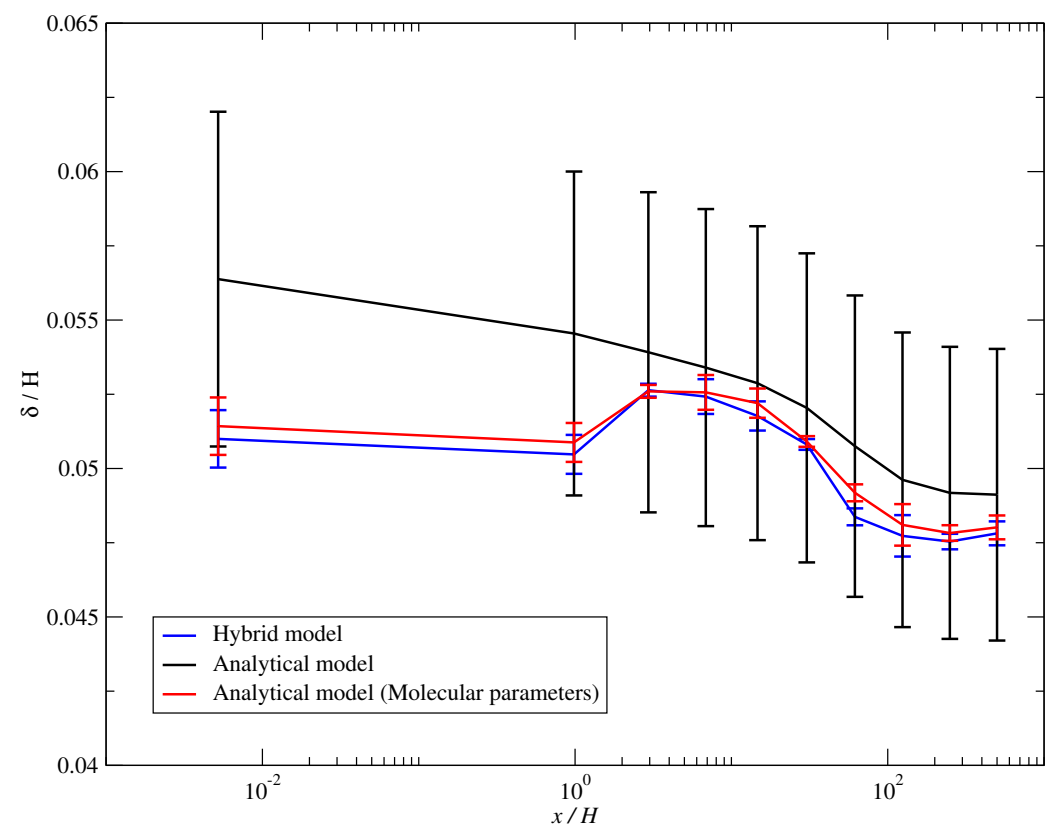

Figure 13: Comparison of the condensate thicknesses, obtained at the 10 molecular block abscissas, between the multiscale hybrid numerical model (blue line) and the multiscale hybrid analytical model (black line). The red line shows the solution obtained with the analytical model (14). The error bars result from the molecular simulations, and are defined by $\left[\min _{i} \delta_{i} ; \max _{i} \delta_{i}\right]$ where $\delta_{i}$ is stemming from three methods: slope change in i) the temperature profiles, ii) the velocity profiles, and iii) an hyperbolic tangent fit of the density profiles with Eq. (11). For the analytical model (black line), 10 percent variation of the physical properties and thermal slip length introduces 20 percent of uncertainties (black error bars).

Figure 14 presents the variation of $T\left(x_{M_{i}}, z=0^{+}\right)$and $T_{l / g}\left(x_{M_{i}}\right)$ as a function of the temperature jumps $T\left(x_{M_{i}}, z=0^{+}\right)$on each molecular block $M_{i}$. The more the fluid goes deeper in the channel, the more it accommodates to the wall temperature. The decrease in the fluid temperature, accompanied by a quicker decrease in the phase change temperature, is also observed. Except for the first block, the heat flux at the wall $\left.(\partial T / \partial z)\right|_{z=0^{+}}$is almost constant all along the channel (Fig. 12(b)), says $q_{0}$. As it is reasonable to consider that the heat transfer coefficient remains constant over the temperature range covered by $T\left(x, z=0^{+}\right)$, namely $\lambda=\lambda_{0}$, the decrease in the temperature difference $T_{l / g}(x)-T\left(x, z=0^{+}\right)$of the liquid leads necessarily 


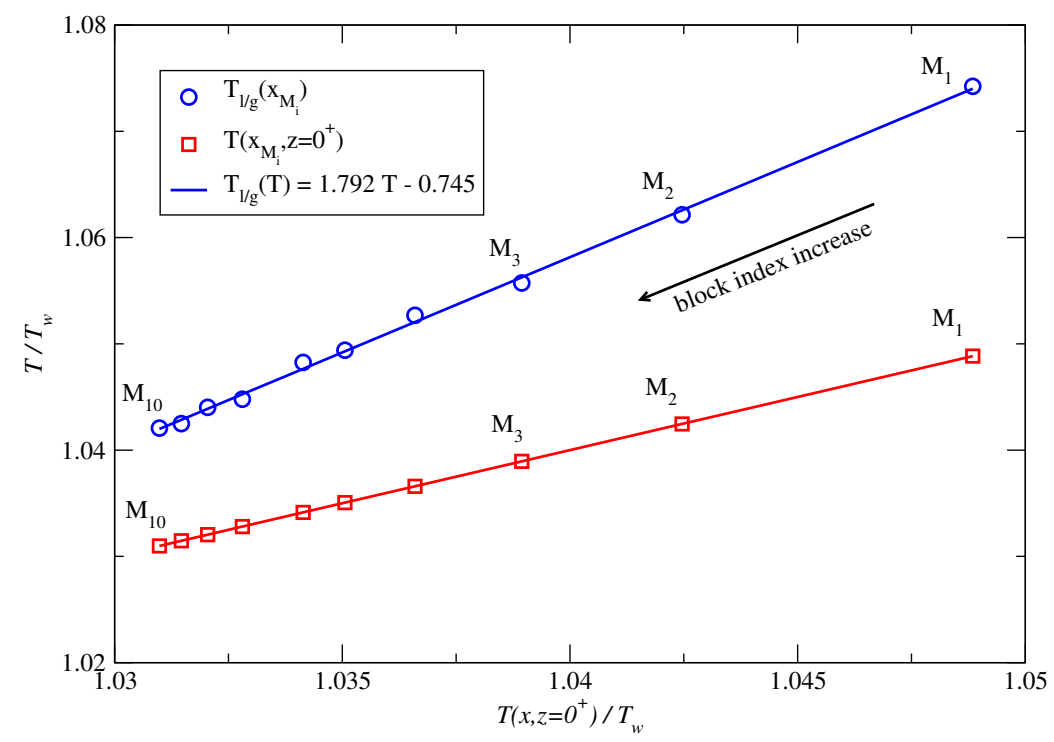

Figure 14: Variations of the temperature jump $T\left(x, z=0^{+}\right)$and phase change temperature $T_{l / g}(x)$ as a function of $T\left(x, z=0^{+}\right)$. The block index increases from right to left.

to the decrease in the condensate film thickness since $\delta(x) \approx \lambda_{0}\left(T_{l / g}(x)-T\left(x, z=0^{+}\right)\right) / q_{0}$.

\section{Conclusion}

The hybrid atomistic-continuum multiscale method presented in Vu et al. (2016) has been extended to deal with flows and heat transfer in long narrow planar channels, when the fluid density is no longer uniform in the cross-sections. The principle of this method consists in domain decomposition, with the fluid/solid interface discretized into a small number of molecular blocks. Each of them is connected to a continuous representation of the bulk through an overlap region which ensures the synchronization and the continuity of the macroscopic variables between the two domains.

In addition to the velocity and temperature coupling between the Finite Volume method and the Molecular Dynamics, a control algorithm of the molecular density has been developed. The adjustment of the parameters and the accuracy of this method have been studied for a Lennard-Jones fluid on a single molecular block, for a fluid flow subjected to phase change in the vicinity of the solid wall.

Then, the new hybrid atomistic/continuum multiscale method has been applied to simulate, first a compressible gas flow, and then a condensation problem in long narrow channel. The influence of the number of blocks and their distribution all along the channel wall has been studied at a low computation cost by substituting the numerous expensive molecular simulations, carried out with the hybrid multiscale method, for suitable analytical temperature and velocity functions. The converged numerical solutions have then served to initialize the molecular dynamics in the different molecular blocks. After this preliminary study, the real hybrid atomistic-continuum multiscale simulations have been carried out. The results issued from the present paper are shown to be satisfactory, capturing the phase change phenomenon, in particular the formation of liquid film near the wall. Although improvements are still necessary, for example to manage automatically the molecular blocks, this numerical method currently devoted to solve the multiscale fluid flow and heat transfer in long narrow channel seems really promising.

\section{Acknowledgements}

This work has benefited from a French government grant managed by ANR within the frame of the national program Investments for the Future ANR-11-LABX-022-01. The authors also thank the Institute for Development and Resources in Intensive Scientific Computing (IDRIS/CNRS) for their support for the project i20142b7277.

\section{References}

M. Allen, D. Tildesley, Computer Simulation of Liquids (Oxford University Press, New York, 1989)

R.B. Bird, W.E. Stewart, E.N. Lightfoot, Transport Phenomena (John Wiley \& Sons, Inc. 2nd edition, 2006)

M.K. Borg, D.A. Lockerby, J.M. Reese, A hybrid molecular-continuum simulation method for incompressible flows in micro/nanofluidic networks. Microfluid Nanofluid (2013a) 
M.K. Borg, D.A. Lockerby, J.M. Reese, A multiscale method for micro/nano flows of high aspect ratio. Journal of Computational Physics 233, 400-413 (2013b)

M.K. Borg, D.A. Lockerby, J.M. Reese, The FADE mass-stat: A technique for inserting or deleting particles in molecular dynamics simulations. J. Chem. Phys. (2014)

M.K. Borg, D.A. Lockerby, J.M. Reese, A hybrid molecular-continuum method for unsteady compressible multiscale flows. J. Fluid Mech. 768, 388-414 (2015)

M. Bugel, G. Galliéro, Thermal conductivity of the Lennard-Jones fluid: an empirical correlation. Chem. Phys. (2008)

E. Chénier, R. Eymard, O. Touazi, Numerical results using a colocated finite-volume scheme on unstructured grids for incompressible fluid flows. Numer. Heat Tr. B-Fund 49(3), 259-276 (2006)

E. Chénier, R. Eymard, R. Herbin, O. Touazi, Collocated finite volume schemes for the simulation of natural convective flows on unstructured meshes. Int. J. Numer. Methods Fluids 56(11), 2045-2068 (2008)

S.K. Dadzie, J.G. Méolans, Temperature jump and slip velocity calculations from an anisotropic scattering kernel. Physica A: Statistical Mechanics and its Applications 358(2-4), 328-346 (2005)

R. Delgado-Buscalioni, P.V. Coveney, Usher: an algorithm for particle insertion in dense fluids. J. Chem. Phys. 119(2), 978-987 (2003)

D. Drikakis, N. Asproulis, Multiscale computational modelling of flow and heat transfer. International Journal of Numerical Methods for Heat \& Fluid Flow (2010)

A. Dupuis, E.M. Kotsalis, P. Koumoutsakos, Coupling lattice boltzmann and molecular dynamics models for dense fluids. Phys. Rev. E 75, 046704 (2007)

G. Galliéro, C. Boned, A. Baylaucq, Molecular dynamics study of the lennard-jones fluid viscosity: Application to real fluids. Ind. Eng. Chem. Res (2005)

N.G. Hadjiconstantinou, Hybrid atomistic-continuum formulations and the moving contact-line problem. J. Comput. Phys. $\mathbf{1 5 4}(2), 245-265$ (1999)

J. Kolafa, I. Nezbeda, The Lennard-Jones fluid: An accurate analytic and theoretically-based equation of state. Fluid Phase Equilib. 100, 1-34 (1994)

J. Liu, S. Chen, X. Nie, M.O. Robbins, A continuum atomistic simulation of heat transfer in micro- and nano-flows. J. Comput. Phys. 227, 279-291 (2007)

S. Maruyama, Molecular dynamics method for microscale heat transfer. Advances in Numerical Heat Transfer 2(6), 189-226 (2000)

S. Maruyama, T. Kimura, A study on thermal resistance over a solid-liquid interface by the molecular dynamics method. Therm. Sci. Eng 7(1), 63-68 (1999)

K. Meier, Computer Simulation and Interpretation of the Transport Coefficients of the Lennard-Jones Model Fluid, PhD thesis, University of the Federal Armed Forces, Hamburg, Germany, 2002

K.M. Mohamed, A.A. Mohamad, A review of the development of hybrid atomistic-continuum methods for dense fluids. Microfluid. Nanofluid. 8(3), 283-302 (2010)

X. Nie, S. Chen, M.O. Robbins, Hybrid continuum-atomistic simulation of singular corner flow. Phys. Fluids 16, 3579-13 $(2004)$

S.T. O'Connell, P.A. Thompson, Molecular dynamics continuum hybrid computations: A tool for studying complex fluid flows. Phys. Rev. E 52, 5792-5795 (1995)

A. Patronis, D.A. Lockerby, M.K. Borg, J.M. Reese, Hybrid continuum-molecular modelling of multiscale internal gas flows. Journal of Computational Physics (2013)

D. Rapaport, The art of molecular dynamics simulation, $2^{\text {nd }}$ edition (Cambridge University Press, New York, 2004)

W. Ren, W. E, Heterogeneous multiscale method for the modeling of complex fluids and micro-fluidics. J. Comput. Phys. 204(1), 1-26 (2005)

D. Stephenson, D.A. Lockerby, M.K. Borg, J.M. Reese, Multiscale simulation of nanofluidic networks of arbitrary complexity. Microfluid Nanofluid (2015)

H. Struchtrup, Maxwell boundary condition and velocity dependent accommodation coefficient. Physics of Fluids 25(11) (2013)

J. Sun, Y.L. He, W.Q. Tao, Molecular dynamics-continuum hybrid simulation for condensation of gas flow in a microchannel. Microfluid. Nanofluid. (2009)

J. Sun, Y.L. He, W.Q. Tao, A molecular dynamics study on heat and mass transfer in condensation over smooth/rough surface. International Journal of Numerical Methods for Heat \& Fluid Flow. 21(2), 244-267 (2011)

J. Sun, Y.L. He, W.Q. Tao, J.W. Rose, H.S. Wang, Multiscale study of liquid flow in micro/nanochannels: effects of surface wettability and topology. Microfluid. Nanofluid. (2012)

R. Tehver, F. Toigo, J. Koplik, J.R. Banavar, Thermal walls in computer simulations. Phys. Rev. E 57(1), 17 (1998)

Q.-D. To, V.-H. Vu, G. Lauriat, C. Léonard, Boundary conditions for gas flow problems from anisotropic scattering kernels. 
Journal of Mathematical Physics 56(10) (2015)

O. Touazi, E. Chénier, R. Eymard, Simulation of natural convection with the collocated clustered finite volume scheme. Computers \& Fluids 37(9), 1138-1147 (2008)

V.H. Vu, B. Trouette, Q.-D. To, E. Chénier, Multiscale modelling and hybrid atomistic-continuum simulation of nonisothermal flows in microchannel. Microfluid. Nanofluid. (2016)

T. Werder, J.H. Walther, P. Koumoutsakos, Hybrid atomistic-continuum method for the simulation of dense fluid flows. J. Comput. Phys. 205(1), 373-390 (2005)

J.L. Xu, Z.Q. Zhou, X.D. Xu, Molecular dynamics simulation of micro-Poiseuille flow for liquid argon in nanoscale. International Journal of Numerical Methods for Heat \& Fluid Flow. 14, 664-688 (2004)

W.J. Zhou, H.B. Luan, Y.L. He, J. Sun, W.Q. Tao, A study on boundary force model used in multiscale simulations with non-periodic boundary condition. Microfluid. Nanofluid. 16, 587-595 (2014) 